ARTICLE

DOI: 10.1038/s41467-017-02680-0 OPEN

\title{
The evolution of substrate discrimination in macrolide antibiotic resistance enzymes
}

Andrew C. Pawlowski ${ }^{1}$, Peter J. Stogios ${ }^{2,3}$, Kalinka Koteva1, Tatiana Skarina ${ }^{2,3}$, Elena Evdokimova ${ }^{2,3}$, Alexei Savchenko $2,3,4 \&$ Gerard D. Wright ${ }^{1}$

The production of antibiotics by microbes in the environment and their use in medicine and agriculture select for existing and emerging resistance. To address this inevitability, prudent development of antibiotic drugs requires careful consideration of resistance evolution. Here, we identify the molecular basis for expanded substrate specificity in Mphl, a macrolide kinase (Mph) that does not confer resistance to erythromycin, in contrast to other known Mphs. Using a combination of phylogenetics, drug-resistance phenotypes, and in vitro enzyme assays, we find that $\mathrm{Mphl}$ and $\mathrm{MphK}$ phosphorylate erythromycin poorly resulting in an antibiotic-sensitive phenotype. Using likelihood reconstruction of ancestral sequences and site-saturation combinatorial mutagenesis, supported by Mph crystal structures, we determine that two non-obvious mutations in combination expand the substrate range. This approach should be applicable for studying the functional evolution of any antibiotic resistance enzyme and for evaluating the evolvability of resistance enzymes to new generations of antibiotic scaffolds.

\footnotetext{
${ }^{1}$ Michael G. DeGroote Institute for Infectious Disease Research and the Department of Biochemistry and Biomedical Sciences, McMaster University, Hamilton, L8S 4L8 ON, Canada. ${ }^{2}$ Department of Chemical Engineering and Applied Chemistry, University of Toronto, Toronto, M5S 3E5 ON, Canada. ${ }^{3}$ Center for Structural Genomics of Infectious Diseases (CSGID), Calgary, T2N 4N1 AB, Canada. ${ }^{4}$ Department of Microbiology, Immunology and Infectious Diseases, University of Calgary, Calgary, T2N 4N1 AB, Canada. Correspondence and requests for materials should be addressed to

G.D.W. (email: wrightge@mcmaster.ca)
} 
A ntibiotic resistance is difficult to overcome because its evolution is persistent and multifactorial ${ }^{1,2}$. In addition to genetic mutations that confer drug insensitivity, mobile genetic elements disseminate resistance genes in pathogens, adding to the complexity and unpredictability of the emergence of resistance in bacterial populations ${ }^{3-5}$. Moreover, the prediction and detection of emerging resistance phenotypes resulting from mutations in resistance genes themselves remains a barrier to clinical surveillance and strategic antibiotic use ${ }^{6-9}$, along with antibiotic drug development ${ }^{10,11}$. A focus of the antibiotic pharmaceutical industry is on identifying new antibiotic scaffolds with decreased susceptibility to current and widespread resistance mechanisms ${ }^{12-15}$. Another fruitful avenue in drug discovery is the development of successive generations of existing antibiotic scaffolds that bypass antibiotic-inactivating enzymes and other resistance mechanisms ${ }^{13,14,16,17}$. For these strategies to be productive in the face of increasing rates of resistance, the functional and evolutionary landscape of resistance enzymes must be interrogated to inform new drug discovery efforts.

Macrolides such as azithromycin are among the most successful and highly prescribed antibiotics in the world. They are first-line treatments for community-acquired respiratory tract infections and gonorrhea ${ }^{18}$, and increasingly used to treat infections caused by multi-drug resistant Enterobacteriaceae ${ }^{19}$. Actinobacteria produce numerous variants of this antibiotic class but clinical implementation is mostly limited to erythromycin and its semi-synthetic derivatives, azithromycin and clarithromycin. The defining features of macrolide antibiotics include a 12-membered to 16-membered macrolactone ring and a dimethylamino sugar linked to the C5 position, which is essential for interacting with its target; ${ }^{20-22}$ the large subunit of the bacterial ribosome (Fig. 1). Some macrolides are decorated with additional sugars at C3 (azithromycin, erythromycin), 4'-OH of the dimethylamino sugar (carbomycin, josamycin), or at multiple positions on the macrolactone (megalomicin, spiramycin, tylosin). Telithromycin is a semi-synthetic derivative of erythromycin and a first generation ketolide with a $\mathrm{C} 11-\mathrm{C} 12$ carbamate and an N-linked alkyl-aryl
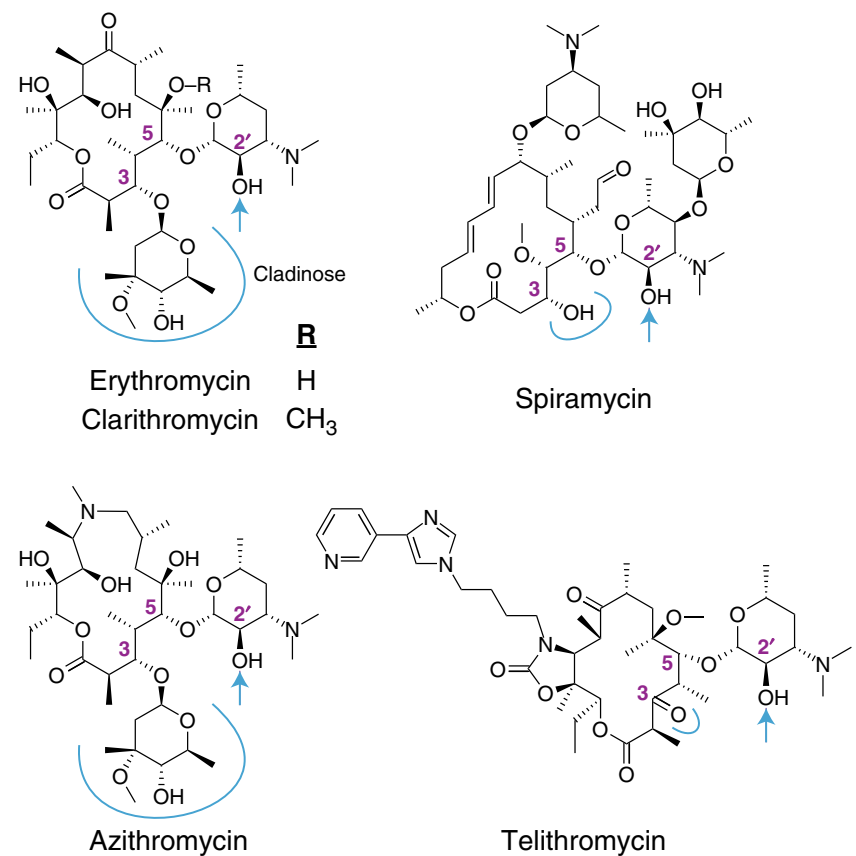

Fig. 1 Structure of macrolide antibiotics. The blue arrow indicates the site of phosphorylation by Mph enzymes. The C3 position is highlighted with a blue line substituent, and a ketone (ketolide) at C3 in place of a cladinose. The inspiration for removing the cladinose in telithromycin development was natural ketolides that bypass the induction of macrolide resistance ${ }^{12}$. Nevertheless, bacteria have evolved to overcome to this modification ${ }^{23-25}$. Macrolide resistance is abundant in pathogenic bacteria, and is most often the result of GTP-dependent macrolide kinases (Mph), ribosomal methyltransferases, or efflux pumps ${ }^{26}$. Mph enzymes inactivate macrolides by phosphorylating the $2^{\prime}-\mathrm{OH}$ of the essential dimethylamino sugar ${ }^{27,28}$, preventing it from binding the ribosome, and providing the chemical rationale for the resistance phenotype.

The Eukaryotic-like kinase superfamily is a structural family related to Eukaryotic protein kinases, and includes antibiotic kinases that modify macrolides (Mph), aminoglycosides ${ }^{29}(\mathrm{APH})$, and tuberactinomycins ${ }^{30}(\mathrm{VPH}, \mathrm{CPH})$. APHs have undergone substantial functional radiation, leading to homologs with different substrate-specificities and regio-specificities ${ }^{31}$. Four Mph homologs $\left(\mathrm{MphA}^{32}, \mathrm{MphB}^{33}, \mathrm{MphC}^{34}, \mathrm{MphE}^{35}\right)$ are mobilized in human pathogens and confer resistance to a wide range of macrolide substrates. In contrast, Mphs from non-pathogenic bacteria are genetically and functionally diverse. We recently described an Mph (MphI) from the environmental bacterium Paenibacillus sp. LC231 that does not confer resistance to macrolides with a C3 cladinose ${ }^{36}$. Paenibacillus sp. LC231 was isolated from Lechuguilla Cave where it was spatiotemporally separated from surface bacteria for over four million years. MphI shares high sequence identity (94\%) to homologs found in related surface Paenibacillus sp., indicating the functional divergence of $\mathrm{MphI}$ is not recent. The Bacillus cereus group have two genetically and functionally distinct Mph enzymes; one that modifies a broad range of macrolides and another that cannot modify macrolides with 16-membered rings ${ }^{37}$. The closest experimentally validated homolog to MphI is MphJ (51\% identity), which confers resistance to erythromycin and tylosin, but not to spiramycin or josamycin ${ }^{38}$. Mph enzymes are therefore adaptable resistance enzymes with complex evolutionary paths.

In this study, we show that narrow range Mphs are common in Bacillales, and their phosphorylation rates of cladinose containing macrolides is insufficient to confer resistance. We reconstruct the evolutionary path to Mph functional divergence using ancestral sequence reconstruction and identify four candidate residues responsible for substrate specificity. Applying phylogenetics, structural biology, protein engineering, and in vitro enzyme assays to the Mph family, we evolve increased substrate range in MphI, which results from multiple non-obvious mutations that, in tandem, increase the catalytic rate towards cladinose containing macrolides. This work demonstrates that while the antibiotic kinases have evolved several distinct functions, there exists significant barriers to acquiring a very related, but non-intrinsic function.

\section{Results}

Multiple independent macrolide kinase mobilization events. Of the $13 \mathrm{Mph}$ homologs that have been experimentally validated for macrolide kinase activity $27,32-37,39-41,7$ are captured by mobile genetic elements. This implies that Mphs are at a high risk for mobilization (Supplementary Fig. 1, Supplementary Table 1). These Mphs share low sequence identity and it is therefore likely that each homolog was mobilized independently; if they originated from a single mobilized $\mathrm{Mph}$, then they should cluster together in phylogenetic analysis. However, only $\mathrm{MphE}, \mathrm{MphF}$, and MphG are phylogenetically clustered, while MphA, MphB, $\mathrm{MphC}$, and $\mathrm{MphN}$ are isolated from other known Mphs (Fig. 2; Supplementary Fig. 2). These results show that Mph genes may 


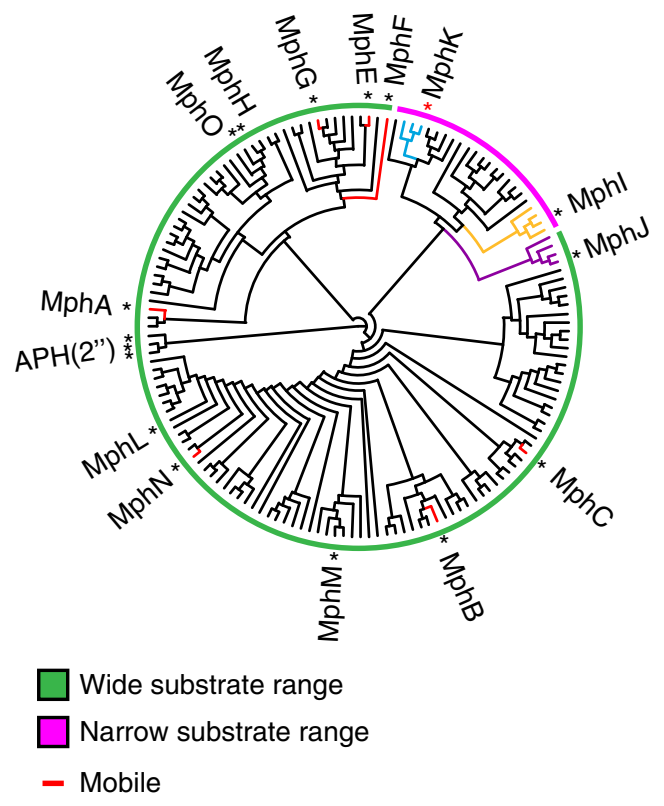

Fig. 2 Phylogenetic reconstruction of macrolide phosphotransferases. Colored bars represent predicted substrate specificity based on phylogenetic clustering of known Mphs and their homologs. Asterisks represent experimentally validated Mphs, and the red asterisk represents MphK, which was identified from this analysis as a Mph that does not confer C3 cladinose macrolide resistance. Colored portions of the phylogenetic tree highlight clusters of interest. Mphs captured on mobile genetic elements are colored red. The tree is presented as a cladogram, and the root drawn with GTP-dependent APH(2") enzymes as outgroups. A cladogram with complete labels and bootstrap values can be found in Supplementary Figure 2

have been captured by mobile genetic elements at least five independent times.

The taxonomic distribution Mphs remains unclear despite several studies detailing their phenotypic, biochemical, and structural characterizations. We expanded the known genetic and taxonomic diversity of Mph enzymes by surveying bacterial strains with genomes deposited in the RefSeq database. Mphs were detected in 4732 genomes across 71 genera, and were predominantly found in Staphylococcus, Acinetobacter, Escherichia, Bacillus, and Klebsiella (Supplementary Fig. 3). Gramnegative pathogens, such as A. baumannii ( $38 \%$ of all $A$. baumannii strains), K. pneumoniae (38\%), and E. coli (13\%) are significantly enriched for Mphs (Fig. 3a). Ancestral A. baumannii, K. pneumoniae, and E. coli strains did not harbor an $\mathrm{Mph}$ homolog, and therefore it is very likely that these were acquired on mobile elements. Analysis of the taxonomic distribution of each Mph homolog reveals that MphA, MphB, and MphE are widespread in Gram-negative bacteria, and that the MphI homologs are limited to Bacillus and Paenibacillus and have not been mobilized (Fig. 3b; Supplementary Fig. 4). Our analysis demonstrates that Mphs are widely distributed across bacterial taxa, and the mobilized homologs are accumulating in Gramnegative pathogens.

Substrate specificity correlates with genetic diversity. MphI is the only known Mph that does not confer C3 cladinose macrolide resistance $^{36}$. If $\mathrm{MphI}$ lost the ability to phosphorylate macrolides with a C3 cladinose, then we would expect it to share a common ancestor with Mphs that have wider substrate specificity, and therefore not positioned near the root of a phylogenetic reconstruction. The closest known homolog to MphI, MphJ (51\%
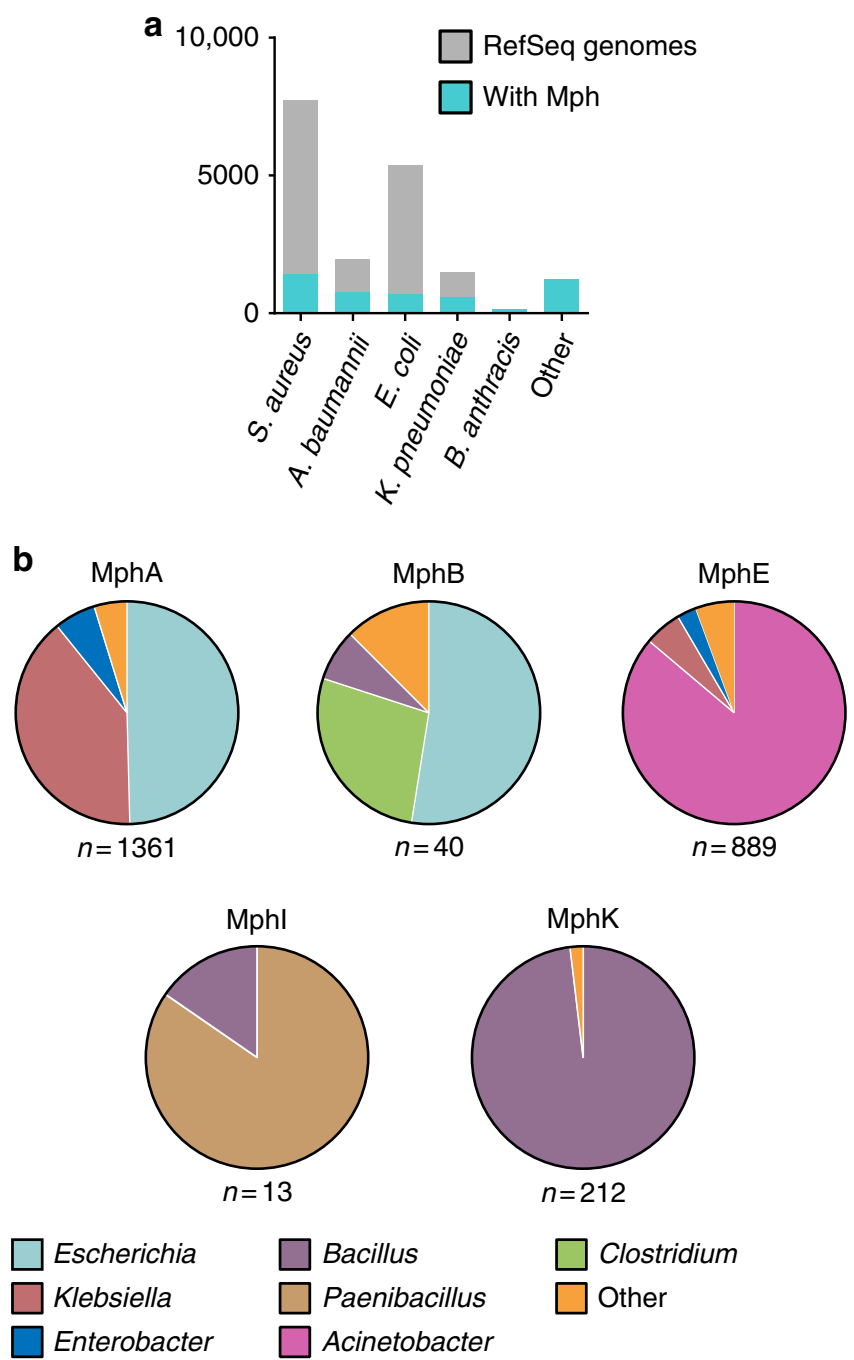

Fig. 3 Taxonomic distribution of Mphs. a The top five bacterial species, in the RefSeq genome database, with Mphs by abundance, and presented in a stacked bar graph. b Taxonomic distribution of MphA, MphB, MphE, Mphl, and MphK by genera

sequence identity), can phosphorylate $\mathrm{C} 3$ cladinose macrolides ${ }^{38}$ ) and is an outgroup to the MphI clade (Fig. 2; Supplementary Fig. 2). The most parsimonious explanation is that MphI lost the ability to phosphorylate C3 cladinose macrolides. Adjacent to MphI is another clade with uncharacterized enzymes from Bacillus that we reasoned may show MphI-like specificity if phylogeny predicts substrate specificity. We heterologously expressed one of the members of this clade in E. coli TOP10 ( $m p h K / y c b J$, 54\% identical to $m p h I$ ) from Bacillus subtilis 168 and verified that it confers resistance to macrolide antibiotics (Supplementary Table 2). Moreover, like $m p h I, m p h K$ does not confer resistance to C3 cladinose macrolides, indicating that Mph substrate specificity correlates with phylogeny.

To further investigate Mph substrate specificity, we performed in vitro enzyme assays with purified MphB, MphI, and MphK. Azithromycin and telithromycin are semi-synthetic derivatives of erythromycin, with telithromycin lacking a C3 cladinose (Fig. 1). MphI and MphK both phosphorylate telithromycin but also phosphorylate azithromycin (Supplementary Fig. 5, Supplementary Table 3), suggesting that resistance phenotype may not correlate with biochemical analysis of drug modification. To confirm this result, we used tandem mass spectrometry to identify the site of phosphorylation on erythromycin, a macrolide 
a

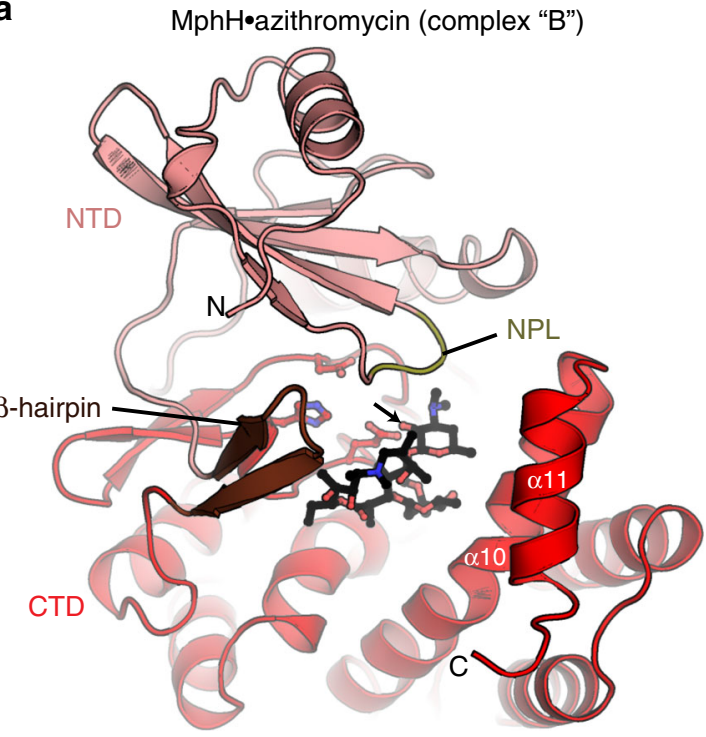

b

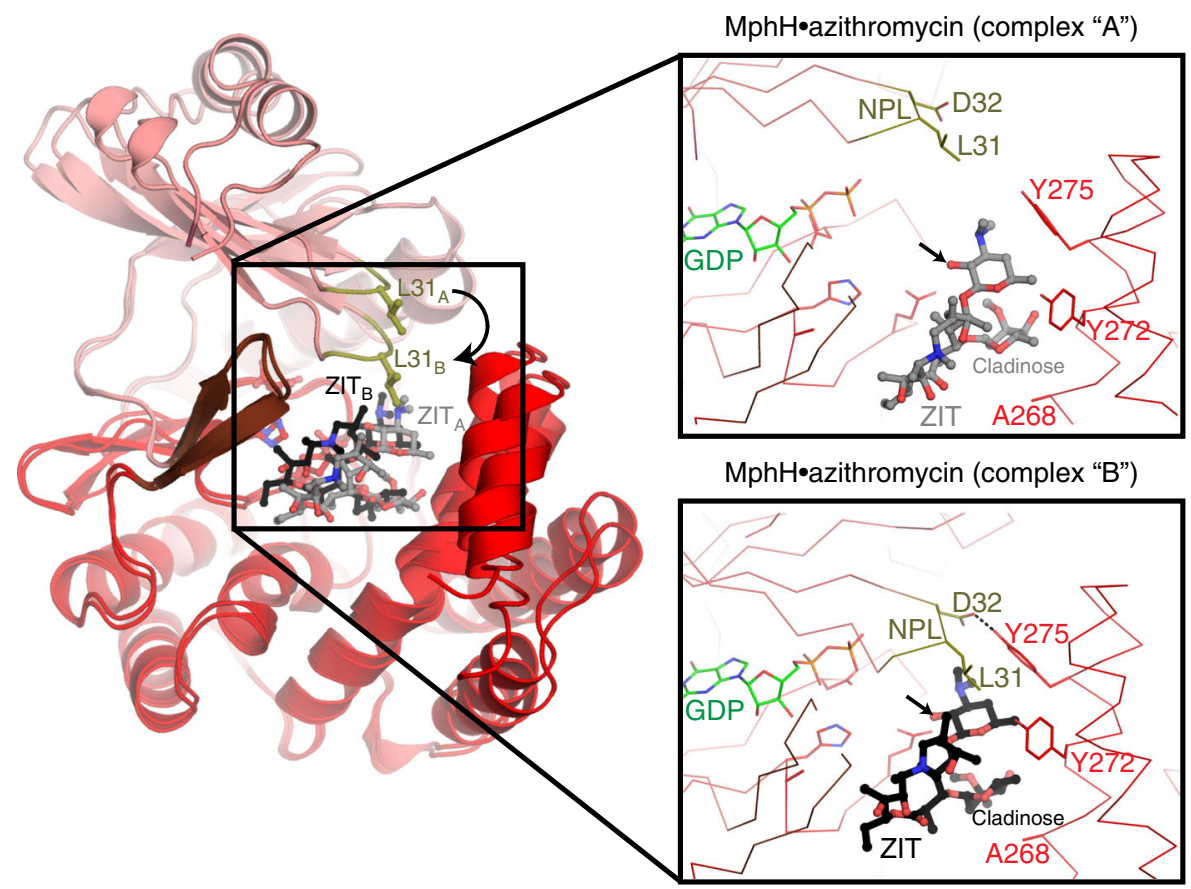

Fig. 4 Crystal structures of $\mathrm{MphH}$. a The MphH•azithromycin (complex "B" of the two complexes in the asymmetric unit of this crystal) is shown in cartoon, with NTD, CTD, NPL, and $\beta$-hairpin regions colored differently. Azithromycin (ZIT) is shown in black ball-and-stick representation. $\alpha 10$ and $\alpha 11$ which make up one face of the macrolide-binding site are labeled. Catalytic residues Glu196, His201, and Asp214 are shown in ball-and-stick. Arrow indicates 2'-OH site of phosphorylation on azithromycin. b Overlay of $\mathrm{MphH} \bullet$ azithromycin complexes " $\mathrm{A}$ " and " $\mathrm{B}$ ", showing conformational differences in NPL (particularly Leu31), position of azithromycin and $\alpha 11$. Inset shows zoom of macrolide-binding sites of each complex, showing key residues that contact the antibiotic compounds. Arrows indicate 2 '-OH site of phosphorylation on azithromycin. GDP is shown from crystal structure of MphH•GDP complex

antibiotic with a similar structure to azithromycin and with wellestablished mass fragmentation patterns ${ }^{42}$. Both MphI and MphK phosphorylate the $2^{\prime}$-OH of erythromycin (Supplementary Fig. 6). Using steady-state kinetics, we demonstrated that MphI and MphK can indeed use C3 cladinose macrolides as substrates, but not efficiently $\left(k_{\text {cat }} / K_{\mathrm{m}} 10^{1}-10^{2}\right)$ (Supplementary Tables 4 and 5). The difference in catalytic constant $\left(k_{\text {cat }} / K_{\mathrm{m}}\right)$ values between $\mathrm{C} 3$ cladinose macrolides and descladinose macrolides is about $10^{3}$ and $10^{1}-10^{3}$ respectively for $\mathrm{MphI}$ and $\mathrm{MphK}$. This poor biochemical specificity translates to the lack of resistance phenotype to these antibiotics when the genes are expressed in E. coli TOP10. To confirm whether this low activity against C3 cladinose macrolides impacts resistance phenotypes, we expressed $m p h I$ and $m p h K$ in an antibiotic hyper-susceptible strain of $E$. coli (E. coli BW25113 $\Delta$ bamB $\Delta$ tol $^{43}$ ). Both $m p h$ genes confer only a modest decrease in sensitivity to these antibiotics due to impaired efflux and outer membrane composition in this strain, verifying that the enzymatic activity observed in vitro has minimal impact on resistance phenotype (Supplementary Table 6).

$\mathrm{MphB}$ is another homolog also reported to have a narrow substrate range, modifying erythromycin but not azithromycin 44 . In contrast to previous reports, we found that $\mathrm{MphB}$ confers resistance to all macrolides tested (Supplementary Tables 2 and 3), and inactivates both telithromycin and azithromycin 
C Position $\quad 40 \quad \underline{111} \quad \underline{166} \quad \underline{211}$

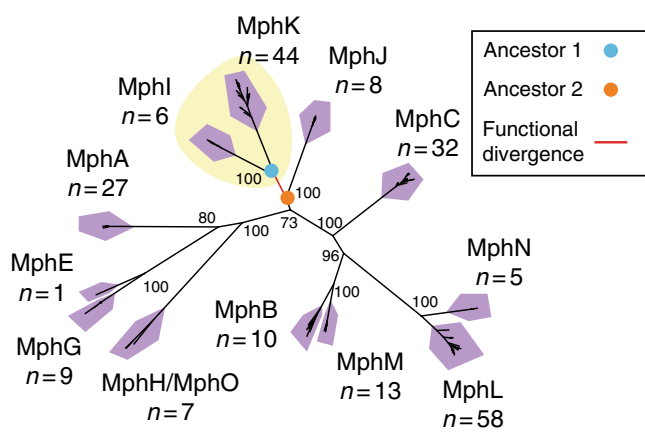

\begin{tabular}{|c|c|c|c|c|}
\hline \multicolumn{5}{|c|}{ Wide substrate range } \\
\hline MphB & $L$ & $\mathrm{~T}$ & M & G \\
\hline $\mathrm{MphH}$ & L & $\mathrm{T}$ & W & A \\
\hline MphJ & L & $\mathrm{T}$ & $\mathrm{T}$ & G \\
\hline Ancestor 2 & $\mathrm{~L}$ & $\mathrm{~T}$ & $\mathrm{M}$ & G \\
\hline \multicolumn{5}{|c|}{ Narrow substrate range } \\
\hline MphK & M & G & T & $\mathrm{P}$ \\
\hline Mphl & M & $S$ & $\mathrm{~T}$ & $\mathrm{P}$ \\
\hline Ancestor 1 & $\mathrm{M}$ & S & $\mathrm{T}$ & $\mathrm{P}$ \\
\hline
\end{tabular}

d

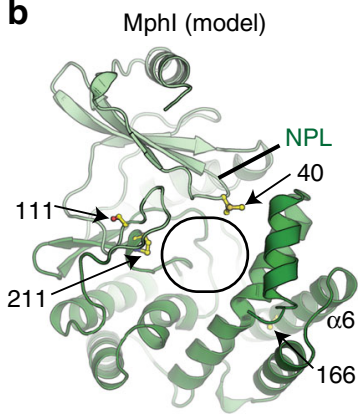

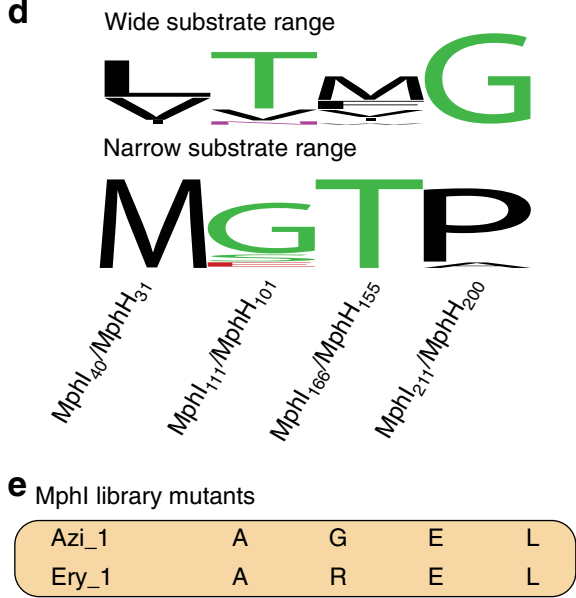

Fig. 5 Ancestral sequence reconstruction of narrow-substrate and wide-substrate range Mphs for identifying possible determinants of substrate specificity. a Closely related homologs ( $>80 \%$ identity) to known Mphs were used to construct a phylogenetic tree. The tree is presented as an unrooted radial phylogram. The red tree branch represents the point of functional divergence between Mphs that can phosphorylate C3 cladinose macrolides, and those that cannot. The colored circles represent ancestral nodes with reconstructed sequences used to compare important residues. Bootstrap values are indicated at branch points. Numbers underneath Mph names represent the number of unique sequences in each cluster. The yellow background represents clades that do not efficiently phosphorylate macrolides with $\mathrm{C} 3$ cladinose. b Model of Mphl based on crystal structure of MphH. Oval represents macrolide-binding pocket as determined from $\mathrm{MphH} \bullet$ azithromycin crystal structures. Residues identified by ancestral sequence reconstruction of Mphl-MphK divergence are colored in yellow. c Amino acid residues at select positions of narrow-spectrum and wide-spectrum Mphs, and their reconstructed ancestors. d Conservation of residues across the two Mph functional subtypes. Sequence conservation is displayed as a sequence logo using WebLogo. Wide substrate range includes sequences in all clades except Mphl and MphK; narrow substrate range includes sequences in only Mphl and MphK clades. e Amino acid residues at select positions of Mphl library mutants with expanded substrate range

\begin{tabular}{|c|c|c|c|c|}
\hline Antibiotic & $\begin{array}{l}\text { Empty } \\
\text { vector }\end{array}$ & mphl & azi_1.1 ${ }^{a}$ & ery_t $\boldsymbol{b}^{\mathbf{b}, \mathbf{c}}$ \\
\hline Erythromycin & 0.5 & 1 & 16 & 16 \\
\hline Clarithromycin & 0.5 & 1 & 4 & 4 \\
\hline Azithromycin & 0.063 & 0.25 & $2-4$ & $2-4$ \\
\hline Telithromycin & 0.13 & $>16$ & $>16$ & $>16$ \\
\hline Spiramycin & 2 & 256 & 256 & 256 \\
\hline Tylosin & 2 & 1024 & 1024 & 1024 \\
\hline Josamycin & $2-4$ & $>64$ & $>64$ & $>64$ \\
\hline Kanamycin & 1 & 1 & 1 & 1 \\
\hline
\end{tabular}

(Supplementary Table 6, Supplementary Fig. 5). Using tandem mass spectrometry, we also confirmed that MphB phosphorylates the desosamine $2^{\prime}-\mathrm{OH}$ of erythromycin (Supplementary Fig. 6). Moreover, MphB modifies macrolides with efficiencies comparable to other Mphs $\left(k_{\mathrm{cat}} / K_{\mathrm{m}} 10^{4}-10^{6}\right)$, and the $K_{\mathrm{m}}$ for C3 cladinose macrolides is $19-69 \times$ lower than MphI (Supplementary Table 8). In aggregate, these results show that C3 cladinose macrolides are good substrates for $\mathrm{MphB}$ and other Mphs including $\mathrm{MphA}^{32,44}$ and $\mathrm{MphH}^{27}$ (Supplementary Table 2), but are poor substrates for $\mathrm{MphI}$ and $\mathrm{MphK}$.

Structural analysis of macrolide recognition by Mph enzymes. To better understand the molecular specificity of Mph enzymes against macrolides, we determined the 3-D structure of $\mathrm{MphB}$ as the apoenzyme, and three forms of $\mathrm{MphH}$ : (1) apoenzyme, (2) in complex with GDP, and (3) in complex with azithromycin (Supplementary Table 4 details of X-ray crystallographic statistics). While preparing this manuscript, crystal structures of MphA and MphB were reported in complex with various macrolides, which we have included in our analysis of substrate specificity $^{45}$. We also attempted to crystallize MphI and MphK as apoenzymes, with several GTP analogs, and with spiramycin, tylosin, and telithromycin, but we were unsuccessful.

As expected, the structures of $\mathrm{MphB}$ (from our study) and $\mathrm{MphH}$ showed that the enzymes adopt the bi-lobe kinase fold reminiscent of $\operatorname{APH}\left(2^{\prime \prime}\right)$ enzyme crystal structures, with an $\mathrm{N}$ terminal domain dominated by $\beta$ sheets and a C-terminal domain 

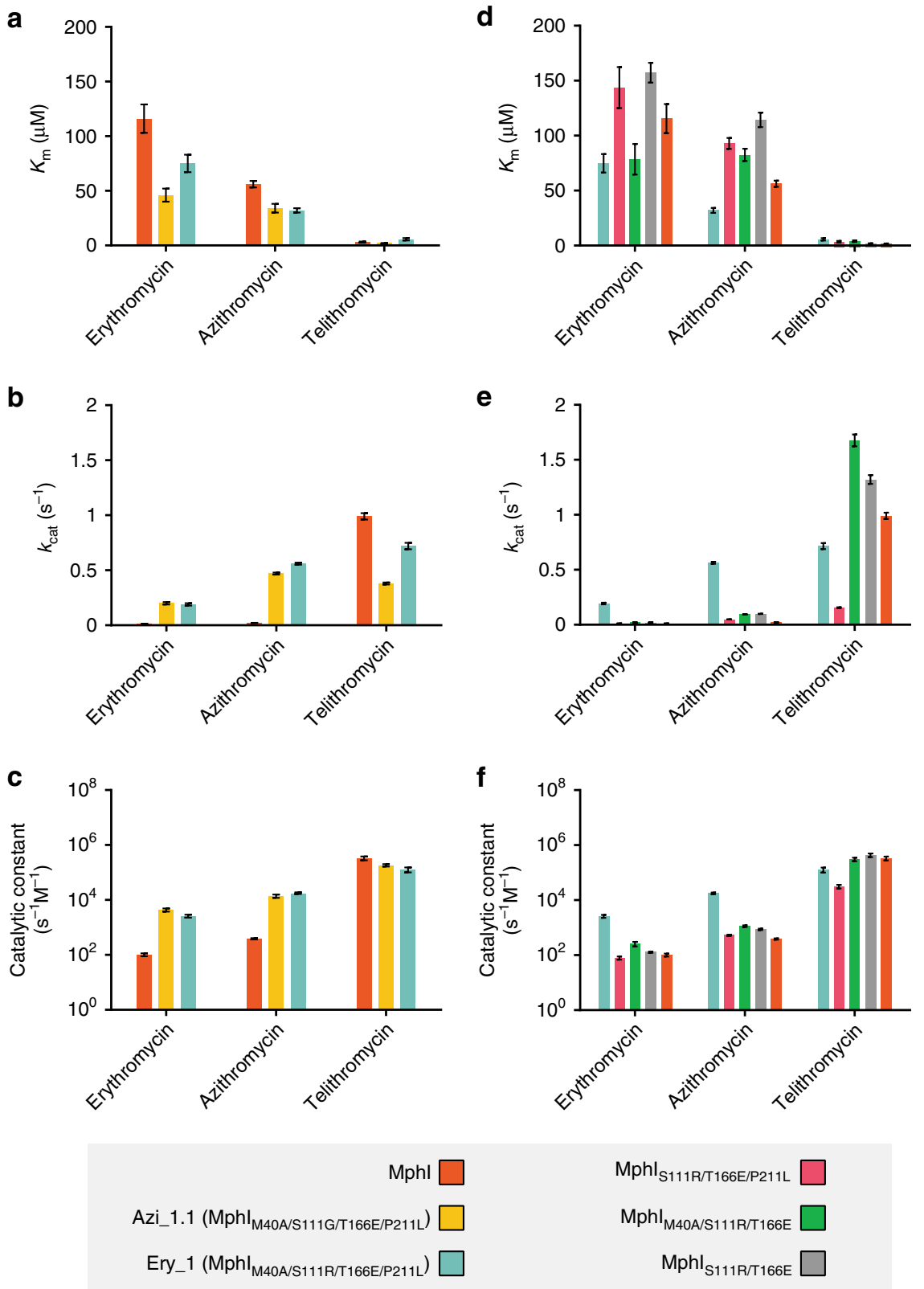

Fig. 6 Kinetic characterization of Mphl and mutants with improved activity towards C3 cladinose macrolides. Steady-state enzyme kinetics for Mphl library mutants $(\mathbf{a}, \mathbf{b}, \mathbf{c})$ and intermediate Ery_1 mutants $(\mathbf{d}, \mathbf{e}, \mathbf{f}) . \mathbf{a} K_{\mathrm{m}}, \mathbf{b} k_{\mathrm{cat}}$, and $\mathbf{c} k_{\mathrm{cat}} / K_{\mathrm{m}}$ values for Mphl and library mutants are presented in clustered bar graph format. $\mathbf{d} K_{\mathrm{m}}, \mathbf{e} k_{\mathrm{cat}}$, and $\mathbf{f} k_{\mathrm{cat}} / K_{\mathrm{m}}$ values for intermediate Ery_1 mutants are also presented. Error bars represent the standard error of the mean (s.e. $\mathrm{m}$.). The $y$-axis for $\mathbf{c}$ and $\mathbf{f}$ are presented in $\log _{10}$ format

comprised mainly of $\alpha$-helices, linked via a $\beta$-hairpin insertion (residues 100-110) (Fig. 4a; Supplementary Fig. 7a).

The asymmetric unit of the $\mathrm{MphH}$ azithromycin crystal contains two copies of the complex ("complex A" and "complex $B$ "), each showing subtle but important conformational differences (Fig. 4b). In both complexes, the azithromycin molecule is bound in a deep, electronegative cleft with one face formed by the end of C-terminal domain (i.e., helices $\alpha 10$ and $\alpha 11$ ), with the other face formed by the apposition of the $\beta$ hairpin, the highly conserved region of the C-terminal domain containing the catalytic residues, and the nucleotide-positioning loop (NPL; residues 29-33) of the N-terminal domain (Fig. 4a). However, it was evident that the "complex B" encloses the bound macrolide more intimately and more appropriately for catalysis (Fig. 4b) and this complex dominates our analysis.
We compared the overall structure of $\mathrm{MphH}$ with $\mathrm{MphA}$ $\left(5 \mathrm{IGI}^{45}\right.$ ) and $\mathrm{MphB}$ (from ref. ${ }^{45}, 5 \mathrm{IGV}$ ) to identify possible conformational changes associated with macrolide binding. While the MphA, MphB, and MphH structures are very similar, we observed structural distinctions in all three enzymes that localized to the (a) NPL, (b) $\beta$-hairpin insert, and (c) multiple residues in the macrolide-binding cleft (Supplementary Fig. 7a). As we observed in $\mathrm{MphH}$, the NPL, and $\beta$-hairpin regions of both MphA and MphB adopt different conformations with macrolide binding. Consistent with the flexibility of $\mathrm{MphH}$ in our crystal structure, these observations highlight that conformational changes in these key regions of $\mathrm{Mph}$ enzymes play a role in macrolide substrate binding.

We compared the positioning of bound macrolides among the $\mathrm{Mph}$ enzymes to gain better insight into recognition of the $\mathrm{C} 3$ cladinose ring. The position of azithromycin in the macrolide- 
Table 2 Antibiotic susceptibility of intermediate mutants between Mphl and Mphl $\mathrm{M40A}_{\mathrm{S}} \mathrm{S11R} / \mathrm{T166E} / \mathrm{P211L}$ expressed in E. coli BW25113 $\Delta$ bamB $\Delta$ tolC

\begin{tabular}{|c|c|c|c|c|c|c|c|c|}
\hline Antibiotic & $\begin{array}{l}\text { ery_1 (Mphl M40A/ } \\
\text { S111R/T166E/P211L) }\end{array}$ & $\begin{array}{l}\text { mphls111R/ } \\
\text { T166E/P211L }\end{array}$ & $\begin{array}{l}\text { mph/ }{ }_{\text {M40A/ }} \\
\text { T166E/P211L }\end{array}$ & $\begin{array}{l}\text { mphI/M40A/ } \\
\text { S111R/P211L }\end{array}$ & $\begin{array}{l}\text { mphl } \\
\text { S111R/T166E }\end{array}$ & $\begin{array}{l}\text { mphl } I_{S 111 R /} \\
\text { T166E }\end{array}$ & $\begin{array}{l}\text { mph/M40A/ } \\
\text { P211L }\end{array}$ & $m p h l^{\mathrm{a}}$ \\
\hline Erythromycin & 32 & 4 & 32 & 32 & 4 & $2-4$ & 32 & $2-4$ \\
\hline Clarithromycin & 8 & 1 & 8 & 8 & 2 & 1 & 16 & 1 \\
\hline Azithromycin & 8 & 1 & 8 & 8 & 2 & 0.5 & $4-8$ & 0.5 \\
\hline Telithromycin & $>16$ & $>16$ & $>16$ & $>16$ & $>16$ & $>16$ & 32 & $>16$ \\
\hline Spiramycin & 256 & 256 & 256 & 256 & 256 & 256 & $128-256$ & $>256$ \\
\hline Tylosin & 1024 & 1024 & 1024 & 1024 & 1024 & $>1024$ & $>1024$ & $>1024$ \\
\hline Josamycin & $>64$ & $>64$ & $>64$ & $>64$ & $>64$ & $>64$ & $>64$ & $>64$ \\
\hline Kanamycin & 1 & 1 & 1 & 1 & 1 & $1-2$ & 1 & 1 \\
\hline
\end{tabular}

a Mphl mutant Ery_1 was found to contain mutations in the promoter region, potentially altering expression and resistance phenotype. Mphl was subcloned into the Ery_ 1 vector and therefore MICs in this table are directly comparable.

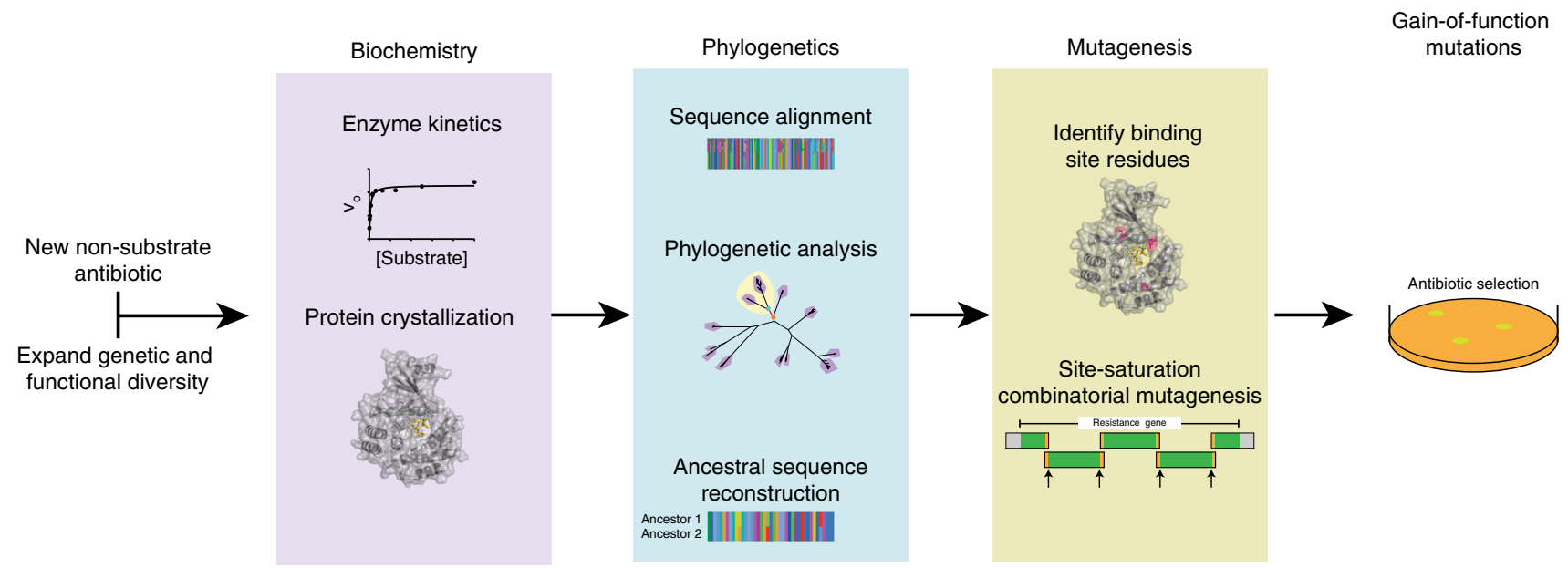

Fig. 7 Workflow for assessing the evolvability of resistance enzymes to new antibiotics using biochemistry, phylogenetics, and mutagenesis. First, expand the known functional diversity of an enzyme class by identifying homologs that include the new antibiotic in its substrate range. Characterize the enzymes using steady-state kinetics and solve the protein structure of at least one homolog. Use the biochemical experiments to correlate functional diversity with phylogenetic diversity for identifying the last common ancestor of each functional subtype (e.g., ancestor of narrow range Mphs and wide range Mphs, respectively). The difference in ancestral protein sequences are residues possibly involved in substrate specificity, which should be prioritized based on proximity to the substrate-binding site in the protein structure. Target these residues for mutagenesis, preferably by saturation mutagenesis at each position, in combination (site-saturation combinatorial mutagenesis). Identify residues expanding substrate specificity by sequencing technologies that generate reads covering the whole gene (e.g., Sanger, Nanopore, PacBio)

binding pockets of MphA, MphB, and $\mathrm{MphH}$ are similar in that the 2' rings are oriented appropriately for catalysis, and the macrolactone ring interacts with the $\beta$-hairpin regions. However, the azithromycin molecule bound to $\mathrm{MphH}$ is positioned $\sim 3 \AA$ closer to the nucleotide binding region as compared to its position in either the MphA or MphB active sites (Supplementary Fig. 7a). Consequently, the C3 cladinose ring of azithromycin occupies deeper regions of the binding clefts of $\mathrm{MphA}$ or $\mathrm{MphB}$. This analysis reveals that the C3 cladinose ring is positioned differently upon binding to individual Mph enzymes.

Substrate selectivity is an emergent property in Mphs. Since structural analysis demonstrated that the $\mathrm{C} 3$ cladinose occupies slightly different positions in the MphA, MphB, and $\mathrm{MphH}$ active sites, we speculated that MphI and MphK may have a large residue facing toward the $\mathrm{C} 3$ cladinose that would sterically hinder binding of these macrolides. We therefore compared the sequence composition of the six residues that approach the cladinose in the Mph structures (i.e., in the MphH azithromycin co-
structure-Glu196, Phe229, Phe265, Ala268, Gly271, Tyr272) across a multiple sequence alignment of all known Mphs and their close homologs ( $>80 \%$ identity). We found no significant correlations between residue type and antibiotic substrate specificity (Fig. 4b; Supplementary Fig. 7b, Supplementary Fig. 8). Ultimately, this analysis of the Mph azithromycin molecular contacts shows that the physiochemical properties of residues in proximity with the $\mathrm{C} 3$ cladinose ring are not conserved even in wide substrate range Mphs. Therefore, C3 cladinose macrolide specificity does not appear to be directed by nearby residues.

Since Mph substrate specificity is a phylogenetic trait, we reasoned that reconstructing the evolutionary path of functional divergence could identify the molecular determinants influencing C3 cladinose phosphorylation ${ }^{46-50}$. To this end, we reconstructed ancestral amino acid sequences ${ }^{51}$ using extant enzymes based on a multiple sequence alignment and phylogeny of related Mph homologs (>80\% identity) (Fig. 5a). Both Mph phylogenies (Figs. 2 and 5a) independently indicate MphI and MphK lost activity towards C3 cladinose macrolides, increasing our confidence in the branching order of Mph sequences. Therefore, 
the equivalent residues different between Ancestor 1 (MphI and $\mathrm{MphK}$ ) and Ancestor 2 (broad substrate range) may be determinants of substrate specificity ${ }^{46}$. We mapped these positions onto the $\mathrm{MphH} \bullet$ azithromycin crystal structure and found four common sites near the macrolide-binding pocket, thus raising the possibility of their involvement in increased activity towards C3 cladinose macrolides (Fig. 5b-d). According to the $\mathrm{MphH} \cdot$ azithromycin structure, one of these sites, $\mathrm{MphH}_{\mathrm{L} 31}$ $\left(\mathrm{MphI}_{\mathrm{M} 40}\right)$ interacts with azithromycin; $\mathrm{MphH}_{\mathrm{L} 31}$ faces toward the desosamine and $\mathrm{C} 6-\mathrm{C} 7$ of the macrolactone ring. $\mathrm{MphH}_{\mathrm{A} 200}$ $\left(\mathrm{MphI}_{\mathrm{P} 211}\right)$ is on the short helix $\alpha 8$ that approaches $\mathrm{MphH}_{\mathrm{Y} 198}$, which forms a key hydrophobic packing interaction with the macrolactone ring close to the methyl on $\mathrm{C} 13$ and the ethyl group on $\mathrm{C} 14 . \mathrm{MphH}_{\mathrm{W} 155}\left(\mathrm{MphI}_{\mathrm{T} 166}\right)$ is in $\alpha 6$ that lines the macrolidebinding pocket and lies within $6 \AA$ of the cladinose. The final site, $\mathrm{MphH}_{\mathrm{T} 101}\left(\mathrm{MphI}_{\mathrm{S} 111}\right)$ is more peripheral to the active site and is in the $\beta$ hairpin but faces away from the macrolide-binding pocket.

We independently mutated each of these positions in MphI to the corresponding residue in the ancestral sequence; none of these variants conferred resistance to C3 cladinose macrolides (Supplementary Table 9). Since engineering new substrate specificity (gain of function) into enzymes is often complex and mutations may require a specific constellation of multiple residues to result in a functional change $\mathrm{e}^{52,53}$, we created an MphI library with mutations at all four positions, in combination, using combinatorial site-saturation mutagenesis. This enabled effective sampling all 160,000 possible amino acid substitutions at each of the four positions identified in ancestral reconstruction, in combination (1.05 million codon combinations). Using the powerful positive selection for antibiotic resistance to screen this mutant library, we were able to identify gain of function azithromycin or erythromycin resistance. We identified three azithromycinresistant variants (Azi_1.1, Azi_1.2, Azi_1.3) with identical amino acid sequences, but different codon combinations, and one erythromycin-resistant variant (Ery_1) (Fig. 5e, Table 1; Supplementary Table 10). All variants with an expanded substrate range were identical at three residue positions: Ala40, Glu166, and Leu211 (mutants of MphI Met40, Thr166, Pro211). This result reveals a very limited number of routes to increase substrate range.

MphI substrate selectivity is correlated with catalytic rate. We purified Azi_1.1 and Ery_1, and verified azithromycin and telithromycin phosphorylation in an in vitro enzyme assay (Supplementary Fig. 5). Mutations in other antibiotic modifying enzymes are known to alter regiospecificity ${ }^{54}$, and the cladinose hydroxyl of azithromycin is a possible phosphate acceptor. We unambiguously identified the desosamine $2^{\prime}-\mathrm{OH}$ as the phosphorylation site of Azi_1.1 and Ery_1 using tandem mass spectrometry (Supplementary Fig. 6). Using steady-state kinetics, we demonstrated that the $k_{\text {cat }} / K_{\mathrm{m}}$ of Azi_1.1 and Ery_1 for C3 cladinose macrolides increased 35-86 fold and 26-46 fold, respectively (Fig. 6a-c; Supplementary Tables 11 and 12). Rather than a significant decrease in $K_{\mathrm{m}}$ for C3 cladinose macrolides, the increase in activity towards these substrates was primarily driven by an increase in $k_{\text {cat }}$. The $K_{\mathrm{m}}$ decreased by about half, while the $k_{\text {cat }}$ value increases $16 \times$ (erythromycin), 22-25×(azithromycin), and $48-51 \times$ (roxithromycin). Our results suggest that the four mutations in Azi_1.1 and Ery_1 increase the rate of product formation, and do not significantly increase catalytically productive C3 cladinose macrolide-binding affinity.

Expanded substrate specificity requires binary mutations. Ancestral sequence reconstruction successfully identified residues implicated in substrate specificity, but the molecular roles of these residues remains unclear. To further explore these, we probed each position's contribution by systematically reversing each Ery_1 (MphI $\left.\mathrm{M}_{\mathrm{M} 0 \mathrm{~A} / \mathrm{S} 111 \mathrm{R} / \mathrm{T} 166 \mathrm{E} / \mathrm{P} 211 \mathrm{~L}}\right)$ mutation to wild-type sequence. $\mathrm{MphI}_{\mathrm{M} 40 \mathrm{~A} / \mathrm{T} 166 \mathrm{E} / \mathrm{P} 211 \mathrm{~L}}$ and $\mathrm{MphI}_{\mathrm{M} 40 \mathrm{~A} / \mathrm{S} 111 \mathrm{R} / \mathrm{P} 211 \mathrm{~L}}$ conferred resistance to $\mathrm{C} 3$ cladinose macrolides and retained the same resistance phenotype as Ery_1 (Table 2). Similarly, $\mathrm{MphI}_{\mathrm{S111R} / \mathrm{T} 166 \mathrm{E}}$ had the same resistance phenotype as MphI. These results demonstrate that positions 111 and 166 are not involved in expanding substrate specificity, consistent with their location more distal to the macrolide-binding site. In contrast, $\mathrm{MphI}_{\mathrm{S} 111 \mathrm{R} / \mathrm{T} 166 \mathrm{E} / \mathrm{P} 211 \mathrm{~L}}$ and $\mathrm{MphI}_{\mathrm{M} 40 \mathrm{~A} / \mathrm{S} 111 \mathrm{R} / \mathrm{T} 166 \mathrm{E}}$ lost the ability to confer C3 cladinose macrolide resistance, indicating that a binary combination of both mutations is required. $\mathrm{MphI}_{\mathrm{M} 40 \mathrm{~A} / \mathrm{P} 211 \mathrm{~L}}$ had the same resistance phenotype as Ery_1 (MphI $\mathrm{M}_{\mathrm{M} 0 \mathrm{~A} / \mathrm{S} 111 \mathrm{R} / \mathrm{T} 166 \mathrm{E} / \mathrm{H}}$ $\mathrm{P} 211 \mathrm{~L}$ ), further supporting the role of both mutations. We confirmed these results using steady-state kinetics of $\mathrm{MphI}_{\mathrm{S} 111 \mathrm{R} / \mathrm{T} 166 \mathrm{E} /}$ P211L, MphI $\mathrm{M}_{\mathrm{M} 0 \mathrm{~A} / \mathrm{S} 111 \mathrm{R} / \mathrm{T} 166 \mathrm{E}}$, and $\mathrm{MphI}_{\mathrm{S} 111 \mathrm{R} / \mathrm{T} 166 \mathrm{E}}$ (Fig. 6d-f; Supplementary Tables 13-15). Each MphI variant had $k_{\text {cat }} / K_{\mathrm{m}}$ values similar to wild-type MphI. These results indicate that together, residues Met40 and Pro211, play key roles in macrolide substrate specificity of Mphs. Met40 and Pro211 are located in the nucleotide-positioning loop and in the highly structurally conserved region near GDP-coordinating/catalytic residues, respectively.

\section{Discussion}

New generations of macrolide antibiotics are being developed to bypass resistance ${ }^{17,55}$, which necessitates understanding the evolutionary landscape of Mphs. In this study, we find that two non-obvious mutations in tandem are required for expanding the resistance phenotype of $\mathrm{MphI}$ to include C3 cladinose macrolides by increasing the catalytic rate. These results are consistent with a plastic MphI active site that accommodates a wide selection of macrolides, but selectively phosphorylates macrolides without a cladinose. Narrow range Mphs such as MphI lost the ability to modify C3 cladinose macrolides, likely because of lack of selection by diverse macrolide structures in the local environment accompanied by neutral drift.

Amino acid residues positioned near, but not in, substratebinding sites can impact function by modulating protein dynamics $^{56,57}$. For example, combinations of mutations located outside the $\beta$-lactam binding pocket of $\beta$-lactamases expand substrate range by increasing conformational flexibility ${ }^{50,56-58}$. Introduction of each mutation individually does not expand the substrate range of $\beta$-lactamases ${ }^{57}$. In our study, M40A and P211L are located outside the MphI macrolide-binding pocket and both mutations are required to confer resistance to $\mathrm{C} 3$ cladinose macrolides. Altogether, the binary M40A and P211L mutations may increase the conformation flexibility of the macrolidebinding pocket, increasing the phosphorylation rate of C3 cladinose macrolides.

Understanding the evolution of resistance enzymes is of considerable interest because of their wide functional and genetic diversity, and their biological importance in medicine and microbial ecology $y^{2,59,60}$. Combining random mutagenesis strategies with antibiotic selection is powerful for interrogating the genetic-phenotypic continuum of resistance enzymes. Indeed, many of the strategies for protein engineering and synthetic biology were first developed using resistance enzymes ${ }^{61-65}$ (e.g., TEM $\beta$-lactamase), and provide a strong foundation for biochemical investigations of resistance. Our approach builds on previous strategies by identifying target positions using phylogenetic and evolutionary principals, and sampling random genetic diversity at each of the four positions simultaneously. The 
strategy we report here offers a single step for efficient sitesaturation combinatorial mutagenesis of multiple positions using Gibson Assembly and productive sampling of the mutant library ( $>1$ million variants) using positive antibiotic selection. Our approach successfully identified candidate positions involved in Mph substrate selectivity that were unidentifiable from Mph•macrolide co-structures reported here or by Fong et al. ${ }^{45}$. Despite the macrolactone ring and dimethylamino sugar shared by all macrolides, the M40A and P211L mutations in MphI specifically increase phosphorylation rate of those with a C3 cladinose without affecting catalysis of descladinose macrolides. In the $\mathrm{MphH} \cdot a z i t h r o m y c i n ~ c o-s t r u c t u r e$, the equivalent $\mathrm{MphH}$ positions Leu31 and Ala200 do not interact with the C3 cladinose. Our study illuminates the utility of evolutionary guided mutagenesis for studying non-obvious molecular determinants of substrate selectivity.

Ancestral sequence reconstruction has proven to be a valuable tool in drug discovery 49 , protein engineering ${ }^{46,48}$, and molecular evolution studies ${ }^{47,50,66}$. We believe our approach will be widely applicable for uncovering the molecular basis of functional divergence in antibiotic resistance enzymes. New generations of antibiotic scaffolds are being designed to bypass resistance mechanisms mobilized in pathogens ${ }^{67}$. However, if a homolog in environmental bacteria confers resistance to the new antibiotics, the potential for evolving resistance is high. Use of ancestral sequence reconstruction combined with structural and biochemical studies, as performed here, will be useful for predicting the possibility and feasibility of expanded resistance phenotypes to semi-synthetic antibiotics (Fig. 7).

Recent platforms that synthesize diverse macrolides will enable the discovery of newer generations of resistance-proof antibiotics ${ }^{17,55}$. Our study suggest that macrolide binding may be a precursor to phenotypic resistance in Mphs. Therefore, next generation macrolide antibiotics that bypass Mph-mediated resistance should also be investigated for active site binding, and whether homologs from environmental bacteria confer resistance.

\section{Methods \\ Antibiotics and reagents. All buffers and salts were purchased from BioShop (Burlington, ON, Canada) unless otherwise specified. Macrolide antibiotics, GTP, pyruvate kinase: lactate dehydrogenase (PK:LDH), phosphoenolpyruvate, and $\mathrm{NADH}$ were purchased from Sigma-Aldrich (Oakville, ON, Canada). Molecular biology kits and organic solvents were purchased from Fisher Scientific (Ottawa ON, Canada). Telithromycin was purified from the drug formulation Ketek (400 $\mathrm{mg}$, Sanofi-Aventis US) ${ }^{36}$.}

Phylogenetic reconstruction of macrolide kinases. Diverse Mph sequences were identified with BLASTp ${ }^{68}$ by querying the Mph sequences in Supplementary Table 1 with an e-value cutoff of 1e-30, and the following flags; -max_target_seqs 500 , and -entrez_query 'NOT partial'. Note that Mph enzymes were renamed for consistency with established nomenclature ${ }^{26}$ and to avoid multiple distinct sequences $(<80 \%$ amino acid identity) with the same name. A hit was defined as having at least $50 \%$ sequence identity and an alignment length $>80 \%$ of the query length. Sequences were clustered at $80 \%$ identity using the cluster_smallmem algorithm of uclust ${ }^{69}$, and aligned with MAFFT (L-INS-i method) ${ }^{70} \cdot \operatorname{APH}\left(2^{\prime \prime}\right)$ enzymes are homologs of Mphs ${ }^{71}$, and therefore APH(2")-IIa, APH(2")-IIIa, and $\mathrm{APH}\left(2^{\prime \prime}\right)$-Ie were used as outgroups for rooting the tree. The alignment was then weighted by alignment confidence using the transitive consistency score (TCS) function of T-coffee ${ }^{72}$. RAxML was used to generate a maximum likelihood phylogenetic tree with the GAMMA model of rate heterogeneity and LG empirical base frequencies (-m PROTGAMMAAUTO) and using 100 rapid bootstraps ${ }^{73}$.

Mph cloning and antibiotic susceptibility testing. $m p h B$ was codon optimized, synthetized as a gBlock (IDT) and cloned into pET28a with an N-terminal histidine tag. The codon optimized $m p h B$ sequence is in Supplementary Table 17. $m p h I$ was previously cloned into pET22 $\mathrm{b}^{36}$. pGDP4 is a derivative of pET28a with the multiple-cloning site under the control of the constitutive lac promoter ${ }^{43} . m p h I$ was subcloned into pGDP4 using the $\mathrm{XbaI}$ and $\mathrm{XhoI}$ restriction sites. E. coli was routinely cultured on LB-Lennox (Bioshop) at $37^{\circ} \mathrm{C}$ overnight, and either $100 \mu \mathrm{g} \mathrm{mL}-1$ ampicillin or $50 \mu \mathrm{g} \mathrm{mL}^{-1}$ kanamycin was used for plasmid selection.
E. coli TOP10 (Invitrogen) was used for routine cloning experiments and susceptibility testing of mphs cloned into pGDP4. E. coli BL21(DE3) (Stratagene, USA) was used for protein overexpression experiments. E. coli BW25113 $\Delta$ bamB $\Delta$ tolC is an antibiotic hypersensitive ${ }^{43}$, and was used for susceptibility testing of Mphs cloning into pGDP4. Antibiotic susceptibility testing was performed according to the Clinical and Laboratory Standards Institute guidelines ${ }^{74}$ for determining minimal inhibitory concentration (MIC) in 96-well plates (Sarstedt, Germany) in duplicate. MIC values were reproducible. A saline suspension of $\mathrm{OD}_{600}$ of 0.1 was made from 2-3 colonies of a fresh overnight, and diluted 1:200 into MuellerHinton Broth (BD Biosciences). MIC values were read after stationary incubation at $37^{\circ} \mathrm{C}$ for $24 \mathrm{~h}$. Susceptibility assays were performed at least two independent times.

Protein overexpression and purification. Mphs were purified as described previously for $\mathrm{MphI}^{36}$. E. coli BL21(DE3) was cultured with shaking at $37^{\circ} \mathrm{C}$ in $1 \mathrm{~L}$ of LB-Lennox until an $\mathrm{OD}_{600}$ of $0.5-0.6$, chilled in an ice bath for $20 \mathrm{~min}$, and protein overexpression induced by the addition of $1 \mathrm{mM}$ IPTG and incubation at $17^{\circ} \mathrm{C}$ for $16 \mathrm{~h}$. Cell mass was harvested by centrifuging at $10,000 \times \mathrm{g}$ for $20 \mathrm{~min}$, washed with cold saline, and resuspended in $20 \mathrm{~mL}$ buffer A (50 mM HEPES pH 7.5, $150 \mathrm{mM}$ $\mathrm{NaCl}, 5 \%$ glycerol, $10 \mathrm{mM}$ imidazole). Cell lysis was performed using a One-shot Cell Disruptor (Constant Systems Limited) at 20,000 psi, and cell debris removed after adding $15 \mathrm{~mL}$ buffer A, $5 \mathrm{mg}$ bovine bovine pancreas DNase, and $2.5 \mathrm{mg}$ of bovine pancreas RNase by centrifugation at 50,000 $\times g$ for $45 \mathrm{~min}$. Proteins were purified using $1 \mathrm{~mL} \mathrm{Ni}^{2+}$-nitrilotriacetic acid column (Qiagen) and a linear gradient of $95 \%$ buffer A to $100 \%$ buffer B (buffer A with $250 \mathrm{mM}$ imidazole) over 20 column volumes. Fractions with pure Mph were pooled and desalted into $50 \mathrm{mM}$ HEPES pH 7.5 using a PD-10 desalting column (GE Scientific). Pure enzyme stocks were stored at $4{ }^{\circ} \mathrm{C}$

Characterization of phosphorylated macrolides. The product of $\mathrm{MphB}, \mathrm{MphI}$, MphK, Azi_1.1, and Ery_1 catalyzed reactions were confirmed with LC-MS analysis. Each $100 \mu \mathrm{L}$ reaction consisted of Mph Buffer (50 mM HEPES pH 7.5, $40 \mathrm{mM}$ $\mathrm{KCl}, 10 \mathrm{mM} \mathrm{MgCl}_{2}$ ), $1 \mathrm{mg} \mathrm{mL}^{-1}$ azithromycin or telithromycin, $2 \mathrm{mM} \mathrm{GTP}$, and 125-250 $\mu \mathrm{g}$ of enzyme. Control reactions were prepared identically, but devoid of enzyme. Reactions were incubated at $37^{\circ} \mathrm{C}$ overnight, stopped with the addition of $100 \mu \mathrm{L}$ cold methanol and stored at $-20^{\circ} \mathrm{C}$. Before analysis, samples were centrifuged at $16,000 \times g$ for $10 \mathrm{~min}$. $20 \mu \mathrm{L}$ of each reaction was injected onto an Agilent 1100 Series LC system and a QTRAP LC/MS/MS System (ABSciex) using the following HPLC conditions; isocratic $5 \%$ solvent B $(0.05 \%$ formic acid in acetonitrile), $95 \%$ solvent $\mathrm{A}$ ( $0.05 \%$ formic acid in water) over $1 \mathrm{~min}$, followed by a linear gradient to $97 \% \mathrm{~B}$ over $7 \mathrm{~min}$ at a flow rate of $1 \mathrm{~mL} \mathrm{~min}^{-1}$ and $\mathrm{C} 18$ column (Sunfire, $5 \mu \mathrm{m}, 4.6 \times 50 \mathrm{~mm}$ ).

Structural elucidation of phosphorylated erythromycin. Tandem mass spectrometry was used to identify the site of phosphorylation by MphB, Azi_1.1, and Ery_1. Each $100 \mu \mathrm{L}$ reaction consisted of Mph Buffer, $0.5 \mathrm{mg} \mathrm{mL}^{-1}$ erythromycin, $2 \mathrm{mM}$ GTP, and 125-250 $\mu \mathrm{g}$ enzyme. Reactions were performed as described above. After centrifugation, samples were diluted 1:1000 into 50\% methanol. High resolution electrospray ionization mass spectra were acquired using Agilent 1290 UPLC separation module and qTOF G6550A mass detector in positive ion mode. Fragmentor voltage was set to $365.0 \mathrm{~V}$. Liquid chromatography separation was carried out using Eclipse C18 $(3.5 \mu \mathrm{m}, 2.1 \times 100 \mathrm{~mm})$ column (Agilent Technologies) and the following pump method: at $0 \mathrm{~min} 95 \%$ solvent $\mathrm{A}(0.1 \% \mathrm{v} / \mathrm{v}$ formic acid in water), from 1 to $7 \mathrm{~min}$ up to $97 \%$ solvent B $(0.1 \% \mathrm{v} / \mathrm{v}$ of formic acid in acetonitrile), at a flow rate $0.4 \mathrm{~mL} \mathrm{~min}^{-1}$. Targeted $\mathrm{MS}^{2}$ acquisition mode was used for the fragmentation under 3 different fixed collision induced dissociation (CID) settings: 10,30 and $40 \mathrm{eV}$. Targeted list contained the following $\mathrm{m} / \mathrm{z}: 734.4681$ $\left(\right.$ erythromycin $\left.[\mathrm{M}+\mathrm{H}]^{+}\right)$and $814.4342\left(\mathrm{Mph}\right.$ erythromycin product $\left.[\mathrm{M}+\mathrm{H}]^{+}\right)$. Fragments were identified based on ref. ${ }^{42}$.

Steady-state kinetics of Mphs. Steady-state kinetics for Mphs were measured using the continuous PK/LDH-coupled assay ${ }^{27,32,36,71}$. Reactions were performed in 96-well plates (Nunc, Thermo Scientific) using Spectramax Plus384 (Molecular Devices) in duplicate. Individual reactions were $100 \mu \mathrm{L}$ and contained $50 \mathrm{mM}$ HEPES pH 7.5, $40 \mathrm{mM} \mathrm{KCl}, 10 \mathrm{mM} \mathrm{MgCl}_{2}, 0.2 \mathrm{mM} \mathrm{NADH}, 3.5 \mathrm{mM} \mathrm{PEP}$, and 4.8 $\mathrm{U} \mathrm{PK} / \mathrm{LDH}$ (pyruvate kinase/lactate dehydrogenase). For determining the $K_{\mathrm{m}}$ for GTP, telithromycin was maintained at $100 \mu \mathrm{M}$ and GTP was varied between 0.49 and $250 \mu \mathrm{M}$. For determining the $K_{\mathrm{m}}$ for macrolides, GTP was maintained at 200 $\mu \mathrm{M}$ and macrolides were varied between $0.49 \mu \mathrm{M}$ and $1000 \mu \mathrm{M}$. Enzyme efficiency varied considerably between substrates, and therefore some enzymes were used at different concentrations depending on the substrate in order to produce a linear range suitable for $K_{\mathrm{m}}$ determination. These concentrations were as follows; MphB $140 \mathrm{nM}$ (most substrates), MphB $36 \mathrm{nM}$ (josamycin), MphI $45 \mathrm{nM}$ (descladinose macrolides), MphI 1.44 $\mu \mathrm{M}$ (C3 cladinose macrolides), Azi_1.1 $90 \mathrm{nM}$ (all substrates), Ery_1 (all substrates), MphK $680 \mathrm{nM}$ (all substrates), $\mathrm{MphI}_{\mathrm{S} 111 \mathrm{R} / \mathrm{T} 166 \mathrm{E} / \mathrm{P} 211 \mathrm{~L}}$ $90 \mathrm{nM}$ (descladinose macrolides), $\mathrm{MphI}_{\mathrm{S} 111 \mathrm{R} / \mathrm{T} 166 \mathrm{E} / \mathrm{P} 211 \mathrm{~L}} 1.43 \mu \mathrm{M}$ (C3 cladinose macrolides), $\mathrm{MphI}_{\mathrm{M} 40 \mathrm{~A} / \mathrm{S} 111 \mathrm{R} / \mathrm{T} 166 \mathrm{E}} 22 \mathrm{nM}$ (descladinose macrolides), $\mathrm{MphI}_{\mathrm{M} 40 \mathrm{~A} /}$ S111R/T166E $430 \mathrm{nM}$ (C3 cladinose macrolides), MphI $\mathrm{S}_{111 \mathrm{R} / \mathrm{T} 166 \mathrm{E}} 22 \mathrm{nM}$ (descladinose 
macrolides), $\mathrm{MphI}_{\mathrm{S} 111 \mathrm{R} / \mathrm{T} 166 \mathrm{E}} 1.43 \mu \mathrm{M}$ (C3 cladinose macrolides). Enzyme kinetics experiments were performed at least two independent times.

\section{Ancestral reconstruction of ancestral Mph sequences. Close Mph homologs} were identified with ncbi-blast-2.2.31 + BLASTp ${ }^{68}$ by querying the Mph sequences in Supplementary Table 1, e-value cutoff of 1e-50, -max_target_seqs 500, and -entrez_query 'NOT partial'. A close homolog was defined as having $>80 \%$ identity and an alignment length $>85 \%$ of the query length. Sequences were aligned using MAFFT (L-INS-i) and the BLOSUM45 substitution matrix. The alignment was weighted using the T-coffee TCS function. A maximum likelihood tree was generated with RAxML with the JTT model (PROTGAMAAAUTO) and rapid bootstrap analysis of 100 replicates. Reconstructed ancestral sequences were inferred using an Empirical Bayes approach (PAML 4.9a) with the jones amino acid replacement matrix. For ancestral reconstruction, the RAxML generated tree was rooted at the point of functional divergence and used the unweighted multiple sequence alignment. The ancestral sequences of each Mph subtype (i.e., those that phosphorylate C3 cladinose macrolide efficiently and those that do it poorly) were contrasted, and these positions were mapped onto the $\mathrm{MphH}$ crystal structure to identify residues near the macrolide-binding pocket. The alignment for ancestral reconstruction was also used to identify amino acid conservation for sequence logos.

Construction of Mphl mutant library. We constructed the MphI mutant library by simultaneously introducing 'NNK' codons at amino acid positions $40,111,166$, and 211 using a hierarchical approach based on the Gibson Assembly method ${ }^{75}$. This method assembled 4 PCR fragments; 3 sections of $m p h I$ and another containing part of the gene and the vector. Oligonucleotides were designed to amplify 93-358 $\left(\mathrm{f}_{1}\right)$ and 471-633 $\left(\mathrm{f}_{3}\right)$, and incorporate 'NNK' codons at the target positions with an additional $25 \mathrm{bp}$ on each end for Gibson Assembly. Additionally, 334-495 $\left(f_{3}\right)$ was amplified, as well as the vector, 1-117, and 634-927 $\left(f_{4}\right)$ (Supplementary Fig. 9). PCR fragments were amplified with Phusion Polymerase (Thermo Scientific) and gel purified with $2 \%$ low melting point agarose (Thermo Scientific). Fragments $\mathrm{f}_{1}(266 \mathrm{bp}), \mathrm{f}_{2}(162 \mathrm{bp}), \mathrm{f}_{3}(188 \mathrm{bp})$, and $\mathrm{f}_{4}$ backbone ( $3636 \mathrm{bp}$ ) were assembled in a $20 \mu \mathrm{L}$ Gibson Assembly reaction (performed in triplicate) and incubated at $50{ }^{\circ} \mathrm{C}$ for $1 \mathrm{~h}$. The Gibson reaction contained; $100 \mathrm{ng} \mathrm{f}_{4}$ backbone, 20 $\operatorname{ng~f}_{1}, 12$ ng f $_{2}, 14$ ng f $_{3}, 0.004 \mathrm{U}$ T5 exonuclease (New England Biolabs), $0.025 \mathrm{U}$ Phusion polymerase, $1 \mathrm{U}$ Taq ligase (New England Biolabs), $0.2 \mathrm{mM}$ each dNTP (FroggaBio), $1 \mathrm{mM}$ NAD (Sigma), $100 \mathrm{mM}$ Tris- $\mathrm{HCl} \mathrm{pH} 7.5$ (Bioshop), $10 \mathrm{mM}$ $\mathrm{MgCl}_{2}$ (Fisher), $10 \mathrm{mM}$ DTT (Bioshop), and $125 \mathrm{mg} \mathrm{mL}^{-1}$ PEG-8000 (Sigma). Reactions were pooled, purified using PCR purification kit (Thermo Scientific) and eluted in $20 \mu \mathrm{L}$ water. A volume of $1 \mu \mathrm{L}$ was used to transform three individual $E$. coli Electromax electrocompetent cell (Invitrogen) reactions. Cells were recovered in a total of $3 \mathrm{~mL}$ Super Optimal broth with Catabolite repression (SOC) (Invitrogen) for $1 \mathrm{~h}$ at $37^{\circ} \mathrm{C}$ with shaking. Library titer was estimated by plating $10^{-3}$ and $10^{-4}$ dilutions and determining the CFU mL $\mathrm{mL}^{-1}$ of transformation. Plasmids from 10 random colonies were isolated, sent for Sanger sequencing (Mobix, McMaster University), and verified to contain a unique missense mutation at each of the four target positions. In total, the library contained 3.9 million variants. The rest of the transformations were used to inoculate $100 \mathrm{~mL}$ LB-Lennox with $100 \mu \mathrm{g}$ $\mathrm{mL}^{-1}$ carbenicillin and incubated with shaking for $16 \mathrm{~h}$ at $37^{\circ} \mathrm{C} .50 \mathrm{~mL}$ was centrifuged at $4000 \times g$ for $1 \mathrm{~h}$ at $4^{\circ} \mathrm{C}$, resuspended in $40 \mathrm{~mL}$ of fresh LB-Lennox and $10 \mathrm{~mL}$ of $80 \%$ glycerol, and stored at $-80^{\circ} \mathrm{C}$. The library was diluted to $\mathrm{OD}_{600}$ of 0.25 ( 200 million CFU $\left.\mathrm{mL}^{-1}\right)$ and $250 \mu \mathrm{L}$ was plated $(\sim 51 \times$ the library) on LB agar with $20 \mu \mathrm{g} \mathrm{mL}^{-1}$ azithromycin and $100 \mu \mathrm{g} \mathrm{mL}^{-1}$ carbenicillin. In another experiment, the library was diluted to $\mathrm{OD}_{600}$ of $0.022\left(\sim 17.8\right.$ million $\left.\mathrm{CFU} \mathrm{mL}^{-1}\right)$ and $250 \mu \mathrm{L}$ was plated $\left(\sim 4.6 \times\right.$ the library) on $250 \mu \mathrm{g} \mathrm{mL}^{-1}$ erythromycin and $75 \mu \mathrm{g}$ $\mathrm{mL}^{-1}$ carbenicillin. The concentration of azithromycin and erythromycin was such that E. coli Electromax harboring wild-type $m p h I$ could not grow. Plasmids from azithromycin or erythromycin-resistant mutants were isolated, transformed into $E$. coli TOP10, and confirmed to be resistant to $\mathrm{C} 3$ cladinose macrolides. This verified that the resistance phenotype was encoded on the plasmid and not a chromosomal mutation. Missense mutations were identified by Sanger sequencing (Mobix, McMaster University). For protein purification experiments MphI mutants were subcloned into pET22b.

Site-directed mutagenesis. Oligonucleotides in Supplementary Table 16 were used for site-directed mutagenesis in the following $50 \mu \mathrm{L}$ reaction; $10 \mathrm{ng}$ plasmid, $0.5 \mu \mathrm{M}$ primers, $200 \mu \mathrm{M}$ dNTPs, $1 \times$ HF buffer, $1 \mathrm{U}$ Phusion polymerase. The following thermocycler conditions were used; initial denaturation $98^{\circ} \mathrm{C} 2 \mathrm{~min}, 98^{\circ}$ $\mathrm{C} 10 \mathrm{~s}, 55^{\circ} \mathrm{C} 20 \mathrm{~s}, 72^{\circ} \mathrm{C} 3.75 \mathrm{~min}$ for 16 cycles, and $7 \mathrm{~min}$ final extension at $72^{\circ} \mathrm{C}$. An aliquot of $2 \mu \mathrm{L}$ of FastDigest DpnI (Thermo Scientific) was added and incubated at $37^{\circ} \mathrm{C}$ for $1 \mathrm{~h}$ to remove template DNA. Reactions were cleaned up with PCR purification, and $5 \mu \mathrm{L}$ was used to transform chemically competent $E$. coli TOP10. Plasmids were isolated and sent for Sanger sequencing to confirm the desired mutations. Wild-type MphI was used for individual mutations predicted with ancestral reconstruction. The Ery $1 \mathrm{MphI}$ mutant was used to reverse mutations to wildtype. All mutations were made independently in pET28a-mphI, pET28a-ery_1, pGDP4-mphI, and pGDP4-ery_1.
Taxonomic distribution of Mphs. The RefSeq genome database was used to evaluate the taxonomic distribution of Mphs in bacteria. The assembly report for all 82,640 RefSeq genomes (as of 29 March 2017) was used to correlate nucleotide accession numbers with RefSeq genomes. The identical protein report was acquired for each protein accession from the phylogenetic reconstruction of diverse Mphs using the efetch Entrez function. Strains with a Mph were identified if the nucleotide accession of a Mph was within the list of RefSeq genomes above. Strains with $2 \mathrm{Mphs}$ were normalized to 1 by matching the unique 4 letter prefix of each nucleotide accession number for WGS sequences, or by matching the entire accession number for complete genomes. This was performed using custom python code.

Crystal structure determination of Mphs. For crystallization, Mph genes were subcloned into the pMCSG53 expression vector that codes for a $\mathrm{N}$-terminal $\mathrm{His}_{6}$-tag and a TEV protease cleavage site. Se-Met-substituted versions of $\mathrm{MphB}$ and $\mathrm{MphH}$ were expressed using the standard M9 high-yield growth procedure according to the manufacturer's instructions (Shanghai Medicilon). Crystallization was performed at room temperature using the sitting drop method and $2 \mu \mathrm{L}$ protein or protein:ligand mixture plus $2 \mu \mathrm{L}$ reservoir solution. The $\mathrm{MphH}$ concentration was $10 \mathrm{mg} \mathrm{mL}^{-1}$ and the $\mathrm{MphB}$ concentration was $90 \mathrm{mg} \mathrm{mL}^{-1}$. Se-Met MphB (apoenzyme) was crystallized with reservoir solution $0.2 \mathrm{M}$ calcium acetate, $0.1 \mathrm{M}$ sodium cacodylate $\mathrm{pH}$ 6.5, $18 \%$ (w/v) PEG $8 \mathrm{~K}$. Native MphH (apoenzyme) was crystallized with reservoir solution $2 \mathrm{M}$ ammonium sulfate, $0.1 \mathrm{M}$ Hepes $\mathrm{pH} 7.5$, 2\% (w/v) PEG 400 and 1\% trehalose. Se-Met $\mathrm{MphH} \bullet \mathrm{GDP}$ complex was crystallized with reservoir solution $2 \mathrm{M}$ ammonium sulfate, 0.1 M Hepes pH 7.5, 2\% (w/v) PEG400, 1\% trehalose, $5 \mathrm{mM}$ azithromycin and $5 \mathrm{mM}$ GMPPCP. Native MphH•azithromycin complex was crystallized with reservoir solution $2 \mathrm{M}$ ammonium sulfate, $5 \mathrm{mM}$ azithromycin and $5 \%$ DMSO. All crystals were cryoprotected in paratone oil prior to data collection. Diffraction data for $\mathrm{MphH}$ (native, apoenzyme) was collected at $100 \mathrm{~K}$ using a Rigaku Micromax 007-HF rotating anode and a Rigaku R-AXIS IV ++detector. Diffraction data for MphB (Se-Met, apoenzyme), $\mathrm{MphH} \bullet \mathrm{GDP}$ (Se-Met) and MphH•azithromycin (native) were collected at the Advanced Photon Source, Argonne National Laboratory, Life Sciences Collaborative Access Team beamlines 21-ID-F or 21-ID-G at the selenium absorption edge. Data was processed by HKL-3000 ${ }^{76}$ or XDS ${ }^{77}$ and Aimless $^{78}$. SAD structure determination was completed using Phenix.autosol ${ }^{79}$ and Molecular Replacement for native structures was compiled using Phenix.phaser. Refinement was completed with Phenix.refine and $\mathrm{Coot}^{80}$. All B-factors were refined as isotropic with TLS parameterization. All geometry was verified using Phenix validation tools and the wwPDB server. Electron density maps are in Supplementary Figure 10.

Code availability. The custom python code used to survey taxonomic distribution of Mphs is available from the corresponding author upon request.

Data availability. The crystal structures of Mph enzymes were deposited into the PDB; MphB Se-Met apoenzyme (5UXA), MphB native apoenzyme (5UXB), MphH (native) GDP + Pi complex (5UXC), and $\mathrm{MphH}$ (Se-Met) azithromycin complex (5UXD). Protein accession numbers for all Mph enzymes included in this study are listen in Supplementary Table 1. Other data are available from the corresponding author upon reasonable request.

Received: 7 September 2017 Accepted: 19 December 2017 Published online: 09 January 2018

\section{References}

1. Perry, J. A. \& Wright, G. D. Forces shaping the antibiotic resistome. BioEssays 36, 1179-1184 (2014)

2. Davies, J. \& Davies, D. Origins and evolution of antibiotic resistance. Microbiol. Mol. Biol. Rev. 74, 417-433 (2010).

3. Jia, B. et al. CARD 2017: expansion and model-centric curation of the comprehensive antibiotic resistance database. Nucleic Acids Res. 45, D566-D573 (2017).

4. Gibson, M. K., Forsberg, K. J. \& Dantas, G. Improved annotation of antibiotic resistance determinants reveals microbial resistomes cluster by ecology. ISME J. 9, 207-216 (2015).

5. Crofts, T. S., Gasparrini, A. J. \& Dantas, G. Next-generation approaches to understand and combat the antibiotic resistome. Nat. Rev. Microbiol. 15, 422-434 (2017).

6. Robicsek, A. et al. Fluoroquinolone-modifying enzyme: a new adaptation of a common aminoglycoside acetyltransferase. Nat. Med. 12, 83-88 (2006).

7. Chanal, C. et al. Nucleotide sequences of CAZ-2, CAZ-6, and CAZ-7 betalactamase genes. Antimicrob. Agents Chemother. 36, 1817-1820 (1992).

8. Henquell, C., Chanal, C., Sirot, D., Labia, R. \& Sirot, J. Molecular characterization of nine different types of mutants among 107 inhibitor- 
resistant TEM beta-lactamases from clinical isolates of Escherichia coli. Antimicrob. Agents Chemother. 39, 427-430 (1995).

9. Casin, I. et al. Aminoglycoside 6'-N-acetyltransferase variants of the Ib type with altered substrate profile in clinical isolates of Enterobacter cloacae and Citrobacter freundii. Antimicrob. Agents Chemother. 42, 209-215 (1998).

10. Silver, L. L. Challenges of antibacterial discovery. Clin. Microbiol. Rev. 24, 71-109 (2011)

11. Sommer, M. O. A., Munck, C., Toft-Kehler, R. V. \& Andersson, D. I. Prediction of antibiotic resistance: time for a new preclinical paradigm? Nat. Rev. Micro 15, 689-696 (2017).

12. Bonnefoy, A., Girard, A. M., Agouridas, C. \& Chantot, J. F. Ketolides lack inducibility properties of MLS(B) resistance phenotype. J. Antimicrob. Chemother. 40, 85-90 (1997).

13. Armstrong, E. S. \& Miller, G. H. Combating evolution with intelligent design: the neoglycoside ACHN-490. Curr. Opin. Microbiol. 13, 565-573 (2010).

14. Papp-Wallace, K. M., Endimiani, A., Taracila, M. A. \& Bonomo, R. A. Carbapenems: past, present, and future. Antimicrob. Agents Chemother. 55, 4943-4960 (2011).

15. Sum, P. E. \& Petersen, P. Synthesis and structure-activity relationship of novel glycylcycline derivatives leading to the discovery of GAR-936. Bioorg. Med. Chem. Lett. 9, 1459-1462 (1999).

16. Rolinson, G. N. Forty years of beta-lactam research. J. Antimicrob. Chemother. 41, 589-603 (1998).

17. Fernandes, P., Martens, E. \& Pereira, D. Nature nurtures the design of new semi-synthetic macrolide antibiotics. J. Antibiot. 70, 527-533 (2017).

18. Hicks, L. A. et al. US outpatient antibiotic prescribing variation according to geography, patient population, and provider specialty in 2011. Clin. Infect. Dis. 60, 1308-1316 (2015).

19. Gomes, C. et al. Macrolide resistance mechanisms in Enterobacteriaceae: focus on azithromycin. Crit. Rev. Microbiol. 43, 1-30 (2017).

20. Kannan, K. et al. The general mode of translation inhibition by macrolide antibiotics. Proc. Natl Acad. Sci. USA 111, 15958-15963 (2014).

21. Dunkle, J. A., Xiong, L., Mankin, A. S. \& Cate, J. H. D. Structures of the Escherichia coli ribosome with antibiotics bound near the peptidyl transferase center explain spectra of drug action. Proc. Natl Acad. Sci. USA 107, 17152-17157 (2010)

22. Tu, D., Blaha, G., Moore, P. B. \& Steitz, T. A. Structures of MLSBK antibiotics bound to mutated large ribosomal subunits provide a structural explanation for resistance. Cell 121, 257-270 (2005).

23. Bailey, M., Chettiath, T. \& Mankin, A. S. Induction of $\operatorname{erm}(C)$ expression by noninducing antibiotics. Antimicrob. Agents Chemother. 52, 866-874 (2008).

24. Wolter, N. et al. Telithromycin resistance in Streptococcus pneumoniae is conferred by a deletion in the leader sequence of $\operatorname{erm}(B)$ that increases rRNA methylation. Antimicrob. Agents Chemother. 52, 435-440 (2008).

25. Park, B. \& Min, Y. H. Inducible expression of $\operatorname{erm}(B)$ by the ketolides telithromycin and cethromycin. Int. J. Antimicrob. Agents 46, 226-227 (2015).

26. Roberts, M. C. Update on macrolide-lincosamide-streptogramin, ketolide, and oxazolidinone resistance genes. FEMS Microbiol. Lett. 282, 147-159 (2008).

27. Bhullar, K. et al. Antibiotic resistance is prevalent in an isolated cave microbiome. PLoS ONE 7, e34953 (2012).

28. Ohara, K., Kanda, T. \& Kono, M. Structure of a phosphorylated derivative of oleandomycin, obtained by reaction of oleandomycin with an extract of an erythromycin-resistant strain of Escherichia coli. J. Antibiot. 41, 823-827 (1988).

29. Hon, W. C. et al. Structure of an enzyme required for aminoglycoside antibiotic resistance reveals homology to eukaryotic protein kinases. Cell 89, 887-895 (1997).

30. Skinner, R. H. \& Cundliffe, E. Resistance to the antibiotics viomycin and capreomycin in the Streptomyces species which produce them. J. Gen. Microbiol. 120, 95-104 (1980).

31. Magnet, S. \& Blanchard, J. S. Molecular insights into aminoglycoside action and resistance. Chem. Rev. 105, 477-498 (2005).

32. Shakya, T. \& Wright, G. D. Nucleotide selectivity of antibiotic kinases. Antimicrob. Agents Chemother. 54, 1909-1913 (2010).

33. Noguchi, N., Katayama, J. \& O'Hara, K. Cloning and nucleotide sequence of the mphB gene for macrolide 2'-phosphotransferase II in Escherichia coli. FEMS Microbiol. Lett. 144, 197-202 (1996).

34. Matsuoka, M., Inoue, M., Endo, Y. \& Nakajima, Y. Characteristic expression of three genes, $\operatorname{msr}(\mathrm{A}), \mathrm{mph}(\mathrm{C})$ and $\operatorname{erm}(\mathrm{Y})$, that confer resistance to macrolide antibiotics on Staphylococcus aureus. FEMS Microbiol. Lett. 220, 287-293 (2003).

35. Schluter, A. et al. Erythromycin resistance-conferring plasmid pRSB105, isolated from a sewage treatment plant, harbors a new macrolide resistance determinant, an integron-containing Tn402-like element, and a large region of unknown function. Appl. Environ. Microbiol. 73, 1952-1960 (2007).

36. Pawlowski, A. C. et al. A diverse intrinsic antibiotic resistome from a cave bacterium. Nat. Commun. 7, 13803 (2016).
37. Wang, C. et al. Functional characterization and phylogenetic analysis of acquired and intrinsic macrolide phosphotransferases in the Bacillus cereus group. Environ. Microbiol. 17, 1560-1573 (2015).

38. Pawlowski, A. C., Westman, E. L., Koteva, K., Waglechner, N. \& Wright, G. D. The complex resistomes of Paenibacillaceae reflect diverse antibiotic chemical ecologies. ISME J. https://doi.org/10.1038/s41396-017-0017-5 (2017).

39. Szczepanowski, R., Krahn, I., Bohn, N., Puhler, A. \& Schluter, A. Novel macrolide resistance module carried by the IncP-1beta resistance plasmid pRSB111, isolated from a wastewater treatment plant. Antimicrob. Agents Chemother. 51, 673-678 (2007)

40. Nonaka, L., Maruyama, F., Suzuki, S. \& Masuda, M. Novel macrolide-resistance genes, $m e f(C)$ and $m p h(G)$, carried by plasmids from Vibrio and

Photobacterium isolated from sediment and seawater of a coastal aquaculture site. Lett. Appl. Microbiol. 61, 1-6 (2015).

41. Yang, J. et al. Characterization of a multiresistant mosaic plasmid from a fish farm Sediment Exiguobacterium sp. isolate reveals aggregation of functional clinic-associated antibiotic resistance genes. Appl. Environ. Microbiol. 80, 1482-1488 (2014).

42. Crowe, M. C., Brodbelt, J. S., Goolsby, B. J. \& Hergenrother, P. Characterization of erythromycin analogs by collisional activated dissociation and infrared multiphoton dissociation in a quadrupole ion trap. J. Am. Soc. Mass. Spectrom. 13, 630-649 (2002).

43. Cox, G. et al. A common platform for antibiotic dereplication and adjuvant discovery. Cell Chem. Biol. 24, 98-109 (2017).

44. Chesneau, O., Tsvetkova, K. \& Courvalin, P. Resistance phenotypes conferred by macrolide phosphotransferases. FEMS Microbiol. Lett. 269, 317-322 (2007)

45. Fong, D. H., Burk, D. L., Blanchet, J., Yan, A. Y. \& Berghuis, A. M. Structural basis for kinase-mediated macrolide antibiotic resistance. Structure 25, 750-761 e755 (2017).

46. Chen, F. et al. Reconstructed evolutionary adaptive paths give polymerases accepting reversible terminators for sequencing and SNP detection. Proc. Nat Acad. Sci. USA 107, 1948-1953 (2010).

47. Randall, R. N., Radford, C. E., Roof, K. A., Natarajan, D. K. \& Gaucher, E. A. An experimental phylogeny to benchmark ancestral sequence reconstruction. Nat. Commun. 7, 12847 (2016)

48. Cole, M. F., Cox, V. E., Gratton, K. L. \& Gaucher, E. A. Reconstructing evolutionary adaptive paths for protein engineering. Methods Mol. Biol. 978, 115-125 (2013).

49. Zakas, P. M. et al. Enhancing the pharmaceutical properties of protein drugs by ancestral sequence reconstruction. Nat. Biotechnol. 35, 35-37 (2017).

50. Risso, V. A., Gavira, J. A., Mejia-Carmona, D. F., Gaucher, E. A. \& SanchezRuiz, J. M. Hyperstability and substrate promiscuity in laboratory resurrections of Precambrian beta-lactamases. J. Am. Chem. Soc. 135, 2899-2902 (2013).

51. Yang, Z. PAML: a program package for phylogenetic analysis by maximum likelihood. Comput. Appl. Biosci. 13, 555-556 (1997).

52. Raquet, $X$. et al. Stability of TEM beta-lactamase mutants hydrolyzing third generation cephalosporins. Proteins 23, 63-72 (1995).

53. Jacquier, H. et al. Capturing the mutational landscape of the beta-lactamase TEM-1. Proc. Natl Acad. Sci. USA 110, 13067-13072 (2013).

54. Holbrook, S. Y. \& Garneau-Tsodikova, S. Expanding aminoglycoside resistance enzyme regiospecificity by mutation and truncation. Biochemistry $\mathbf{5 5}$, 5726-5737 (2016).

55. Seiple, I. B. et al. A platform for the discovery of new macrolide antibiotics. Nature 533, 338-345 (2016).

56. Zou, T., Risso, V. A., Gavira, J. A., Sanchez-Ruiz, J. M. \& Ozkan, S. B. Evolution of conformational dynamics determines the conversion of a promiscuous generalist into a specialist enzyme. Mol. Biol. Evol. 32, 132-143 (2015).

57. Gonzalez, M. M., Abriata, L. A., Tomatis, P. E. \& Vila, A. J. Optimization of conformational dynamics in an epistatic evolutionary trajectory. Mol. Biol. Evol. 33, 1768-1776 (2016).

58. Tomatis, P. E. et al. Adaptive protein evolution grants organismal fitness by improving catalysis and flexibility. Proc. Natl Acad. Sci. USA 105, 20605-20610 (2008).

59. Morar, M. \& Wright, G. D. The genomic enzymology of antibiotic resistance. Annu. Rev. Genet. 44, 25-51 (2010).

60. Wright, G. D. The antibiotic resistome: the nexus of chemical and genetic diversity. Nat. Rev. Microbiol. 5, 175-186 (2007).

61. Melnikov, A., Rogov, P., Wang, L., Gnirke, A. \& Mikkelsen, T. S. Comprehensive mutational scanning of a kinase in vivo reveals substratedependent fitness landscapes. Nucleic Acids Res. 42, e112 (2014).

62. Orencia, M. C., Yoon, J. S., Ness, J. E., Stemmer, W. P. \& Stevens, R. C. Predicting the emergence of antibiotic resistance by directed evolution and structural analysis. Nat. Struct. Biol. 8, 238-242 (2001).

63. Stemmer, W. P. Rapid evolution of a protein in vitro by DNA shuffling. Nature 370, 389-391 (1994)

64. Haller, G. et al. Massively parallel single-nucleotide mutagenesis using reversibly terminated inosine. Nat. Methods 13, 923-924 (2016). 
65. Bratulic, S., Gerber, F. \& Wagner, A. Mistranslation drives the evolution of robustness in TEM-1 beta-lactamase. Proc. Natl Acad. Sci. USA 112, 12758-12763 (2015).

66. Gaucher, E. A., Thomson, J. M., Burgan, M. F. \& Benner, S. A. Inferring the palaeoenvironment of ancient bacteria on the basis of resurrected proteins. Nature 425, 285-288 (2003).

67. Pawlowski, A. C., Johnson, J. W. \& Wright, G. D. Evolving medicinal chemistry strategies in antibiotic discovery. Curr. Opin. Biotechnol. 42, 108-117 (2016).

68. Camacho, C. et al. BLAST+: architecture and applications. BMC Bioinformatics 10, 421 (2009).

69. Edgar, R. C. Search and clustering orders of magnitude faster than BLAST. Bioinformatics 26, 2460-2461 (2010).

70. Katoh, K. \& Standley, D. M. MAFFT multiple sequence alignment software version 7: improvements in performance and usability. Mol. Biol. Evol. 30, 772-780 (2013).

71. Shakya, T. et al. A small molecule discrimination map of the antibiotic resistance kinome. Chem. Biol. 18, 1591-4601 (2011).

72. Chang, J. M., Di Tommaso, P. \& Notredame, C. TCS: a new multiple sequence alignment reliability measure to estimate alignment accuracy and improve phylogenetic tree reconstruction. Mol. Biol. Evol. 31, 1625-1637 (2014).

73. Stamatakis, A. RAxML version 8: a tool for phylogenetic analysis and postanalysis of large phylogenies. Bioinformatics 30, 1312-1313 (2014).

74. Clinical and Laboratory Standards Institute. Methods for dilution: antimicrobial susceptibility testing of bacteria that grow aerobically. (CLSI, Wayne, PA, USA, 2012).

75. Gibson, D. G. et al. Enzymatic assembly of DNA molecules up to several hundred kilobases. Nat. Methods 6, 343-345 (2009).

76. Minor, W., Cymborowski, M., Otwinowski, Z. \& Chruszcz, M. HKL-3000: the integration of data reduction and structure solution--from diffraction images to an initial model in minutes. Acta Crystallogr. D Biol. Crystallogr. 62, 859-866 (2006).

77. Kabsch, W. XDS. Acta Crystallogr. D Biol. Crystallogr. 66, 125-132 (2010).

78. Winn, M. D. et al. Overview of the CCP4 suite and current developments. Acta Crystallogr. D Biol. Crystallogr. 67, 235-242 (2011).

79. Adams, P. D. et al. PHENIX: a comprehensive Python-based system for macromolecular structure solution. Acta Crystallogr. D Biol. Crystallogr. 66, 213-221 (2010)

80. Emsley, P. \& Cowtan, K. Coot: model-building tools for molecular graphics. Acta Crystallogr. D Biol. Crystallogr. 60, 2126-2132 (2004).

\section{Acknowledgments}

We thank Nicholas Waglechner (McMaster) for useful discussions on phylogenetics, Mph surveillance, and ancestral sequence reconstruction. We also thank Murray Junop (Western University) for helpful discussion on protein engineering experiments. This research was funded by a Canadian Institutes of Health Research grant (FRN-148463) and by a Canada Research Chair in Antibiotic Biochemistry (to G.D.W.) and through funds from the National Institute of Allergy and Infectious Diseases, National Institutes of Health, Department of Health and Human Services, under Contract No. HHSN272201200026C

\section{Author contributions}

A.C.P. and G.D.W. designed research. A.C.P., P.J.S., and K.K. performed research and analyzed data. A.C.P. performed the following experiments; antibiotic susceptibility testing, phylogenetics, enzyme kinetics, ancestral sequence reconstruction, mass spectrometry, site-saturation combinatorial mutagenesis, and in vitro enzyme assays. P.G.S. performed crystal structure determination of MphH. K.K. performed tandem mass spectrometry experiments. T.S. and E.E. performed protein crystallization. A.S. supervised protein crystallization and contributed to interpreting mechanism of expanded substrate range. A.C.P, P.J.S, and G.D.W wrote the manuscript with input from all authors.

\section{Additional information}

Supplementary Information accompanies this paper at https://doi.org/10.1038/s41467 017-02680-0.

Competing interests: The authors declare no competing financial interests.

Reprints and permission information is available online at http://npg.nature.com/ reprintsandpermissions/

Publisher's note: Springer Nature remains neutral with regard to jurisdictional claims in published maps and institutional affiliations.

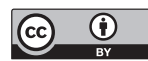

Open Access This article is licensed under a Creative Commons Attribution 4.0 International License, which permits use, sharing, adaptation, distribution and reproduction in any medium or format, as long as you give appropriate credit to the original author(s) and the source, provide a link to the Creative Commons license, and indicate if changes were made. The images or other third party material in this article are included in the article's Creative Commons license, unless indicated otherwise in a credit line to the material. If material is not included in the article's Creative Commons license and your intended use is not permitted by statutory regulation or exceeds the permitted use, you will need to obtain permission directly from the copyright holder. To view a copy of this license, visit http://creativecommons.org/ licenses/by/4.0/

(C) The Author(s) 2017 